\title{
Übersichtsbeitrag
}

\section{Thermische Nutzung von Biomasse und Reststoffen in Deutschland}

\section{Matthias Gaderer ${ }^{1, *}$ und Hartmut Spliethoff ${ }^{1,2}$}

DOI: $10.1002 /$ cite. 201100132

\begin{abstract}
Ein Überblick zum Stand der Technik wird gegeben und neue Entwicklungen auf dem Gebiet der thermischen Nutzung der Biomasse vorgestellt. Die thermische Nutzung von Biomasse in Deutschland ist eine wesentliche Säule der Bereitstellung von Erneuerbaren Energien. Der Primärenergiebedarf und die Potenziale der Biomasse in Deutschland werden dargestellt und mit anderen Regionen verglichen. Es ist ersichtlich, dass eine deutliche Steigerung der Biomassenutzung durch begrenzte Ressourcen limitiert ist. Mit neuen Verfahren zur Biomasseaufbereitung, wie der Torrefizierung und der Hydrothermalen Karbonisierung können weitere biogene Reststoffe zur Nutzung durch Verbrennung und Vergasung erschlossen werden. Dargestellt werden daher der Stand der Verbrennungs- und Vergasungsverfahren und aktuelle Entwicklungen auf dem Gebiet der Synthesegasherstellung.
\end{abstract}

Schlagwörter: Biomasse, Energetische Nutzung, Reststoffe, Verbrennung, Vergasung

Eingegangen: 11. Juli 2011; revidiert: 18. August 2011; akzeptiert: 19. August 2011

\section{Thermal Use of Biomass and Residual Materials in Germany}

The State of the Art and new developments in the area of the thermal use of the biomass is indicated. The thermal use of biomass in Germany is an essential part of the supply with renewable energy. The primary energy demand and the potentials of the biomass in Germany are shown and compared to other regions. It is evident that a clear increase of the biomass use is limited by restricted resources in biomass. However, with new technologies and procedures of the biomass processing and pretreatment other resources can be opened to the use by combustion and gasification. Hence, the state of the combustion and gasification procedures as well as actual developments in the area of the synthesis gas production is shown.

Keywords: Biomass, Combustion, Gasification, Energetic use, Residual materials

\section{Einleitung}

Die Nutzung fester Brennstoffe zur Wärme- und Stromerzeugung ist eine der ältesten Technologien zur Energieversorgung. Die Verbrennung von Abfällen in Deutschland wurde erstmals 1896 in Hamburg durchgeführt und war englischen Anlagen nachempfunden. Bereits damals ver-

\footnotetext{
${ }^{1}$ Dr. Matthias Gaderer (gaderer@tum.de), Prof. Hartmut Spliethoff, Technischen Universität München, Lehrstuhl für Energiesysteme, Boltzmannstraße 15, 85748 Garching, Germany; ${ }^{2}$ Prof. Hartmut Spliethoff, ZAE Bayern, Walther-Meißner-Straße 6, 85748 Garching, Germany.
}

wendete man Roste mit einer Zuführung der Verbrennungsluft von unten [1]. Erste Kohlekraftwerke entstanden ebenfalls zu dieser Zeit. Edison baute in den USA 1882 das erste Kohlekraftwerk zur Elektrifizierung von New York [2]. In Deutschland wurde die Ära der Kohlekraftwerke um 1914 mit dem Bau eines Kraftwerkes in Weisweiler in der Nähe von Köln eingeleitet. Biomasse hatte $\mathrm{zu}$ der Zeit für die Stromerzeugung noch keine große Bedeutung. Heute ist die Verbrennung fester Brennstoffe im Dampfkraftwerk die Standardtechnologie zur Stromerzeugung. Kohle ist weltweit mit etwa $41 \%$ der wichtigste Energieträger zur Stromerzeugung [3, 4].

Ein Vergleich in Tab. 1 zeigt, dass weltweit 37\% der Primärenergie mit festen Brennstoffen bereitgestellt werden. In China ist die Bedeutung der festen Brennstoffe mit $76 \%$ 
Tabelle 1. Die Deckung der benötigten Primärenergie im Jahr 2007 [5].

\begin{tabular}{lrrrr}
\hline Ressourcen & Welt & EU-27 & USA & China \\
\hline Kohle [\%] & 26,5 & 18,8 & 23,7 & 65,6 \\
Erdöl [\%] & 34,1 & 34,5 & 39,0 & 18,2 \\
Erdgas [\%] & 20,9 & 24,6 & 23,0 & 3,1 \\
Nuklearenergie [\%] & 5,9 & 13,9 & 9,3 & 0,8 \\
Wasserkraft [\%] & 2,2 & 1,5 & 0,9 & 2,1 \\
Biomasse und Abfall [\%] & 9,8 & 5,7 &, 5 & 9,9 \\
andere Erneuerbare & 0,6 & 0,9 & 0,6 & 0,3 \\
Energien [\%] & 100 & 100 & 100 & 100 \\
Summe [\%] & 502,9 & 73,6 & 97,8 & 82,5 \\
Summe [EJ] & & & &
\end{tabular}

besonders hoch. Der derzeitige Anteil der Biomasse beträgt je nach Region 4-10\%. In vielen Gebieten, wie Asien, Afrika und Südamerika wird dieser Anteil jedoch überwiegend durch die Nutzung von Biomasse mit relativ einfachen Technologien im Haushaltsbereich und für Heizzwecke erreicht.

Die energetische Nutzung der Biomasse wurde in den letzten Jahrzehnten sehr stark von europäischen Ländern mit einer ausgeprägten Holzindustrie vorangetrieben. Beispiele dafür sind die Länder Schweden, Finnland und Österreich.

In Deutschland deckten die festen Brennstoffe im Jahr 2009 ca. $26 \%$ des Primärenergiebedarfes. Gemäß Tab. 2 hat die Kohle einen Anteil von 22\%, Holzbrennstoffe 3,3\%, Müll und Deponiegase 0,7\%. Während der Anteil aus Kohle in Deutschland abnimmt - der Wert betrug 1992 noch ca. 30\% - und der Wert für Abfälle nahezu konstant blieb, stieg der Wert für Biomasse von 0,4\% im Jahr 1992 kontinuierlich an und hat sich ausgehend von diesem niedrigen Wert vervielfacht. Holzbrennstoffe, Biogas und Bioöle zusammen decken derzeit rund $6 \%$ des energetischen Primärenergiebedarfs und 4,6\% des Strombedarfs [6]. Somit stellt Biomasse einen der wichtigsten erneuerbaren Energieträger dar.

Aufgrund der zunehmenden Nachfrage nach Biomasse steigen die Preise für Energieholz. Die Folge ist, dass alternative günstigere Biomassen wie biogene Abfälle an Bedeutung gewinnen und kostenintensive Energiepflanzen konkurrenzfähig werden.

Aktuelle Szenarien der IEA (International Energy Agency) [5] gehen von einem starken Anstieg der Biomassenutzung aus. Der Beitrag der Biomasse zur Primärenergiedeckung soll von $10 \%$ in 2007 auf $16 \%$ in 2030 ansteigen. Die Umwandlung der Biomasse kann in modernen dezentralen Anlagen mit Kraft-Wärmehen.
Tabelle 2. Deckung des Primärenergiebedarfs (PE) und der Stromerzeugung in Deutschland im Jahr 2010 [6].

\begin{tabular}{lrr}
\hline Ressourcen & PE & Strom \\
\hline Wasserkraft [\%] & 0,5 & 4,33 \\
Windkraft (2010) [\%] & 0,96 & 5,8 \\
Photovoltaik (2010) [\%] & 0,31 & 1,9 \\
Holz [\%] & 3,7 & $4,6 *$ \\
Bioöle [\%] & 1,3 & $-*$ \\
Klärgas, Biogas [\%] & 1,7 & $-*$ \\
Müll und Deponiegas [\%] & 0,76 & 1,0 \\
Solar, Geothermie, Abwärme [\%] & 0,28 & 0,004 \\
Mineralöl [\%] & 33,6 & 1,3 \\
Steinkohle [\%] & 12,1 & 18,8 \\
Braunkohle [\%] & 10,7 & 23,2 \\
Erdgas [\%] & 21,8 & 13,4 \\
Kernenergie [\%] & 10,9 & 22,5 \\
Sonstige [\%] & 1,4 & 3,17 \\
\hline Summe [\%] & 100 & 100 \\
Summe [PJ] & 14057 & 2249 \\
\hline
\end{tabular}

*Biomasse gesamt (Holz, Bioöle, Klärgas einschl. Biogas).

Kopplung, durch Mitverbrennung in Kohlekraftwerken und durch die Herstellung von flüssigen und gasförmigen Energieträgern erfolgen. Insbesondere die Nutzung der Biomasse zur Stromgewinnung soll weltweit erheblich ansteigen. Je nach Szenario wird für die Stromerzeugung aus Biomasse von einer Vervielfachung um den Faktor 3-5 gegenüber dem Jahr 2007 ausgegangen (Abb. 1).

Um die Klimaschutzziele zu erreichen, wurden auf EUEbene im Rahmen der Renewable Energy Directive (RED)

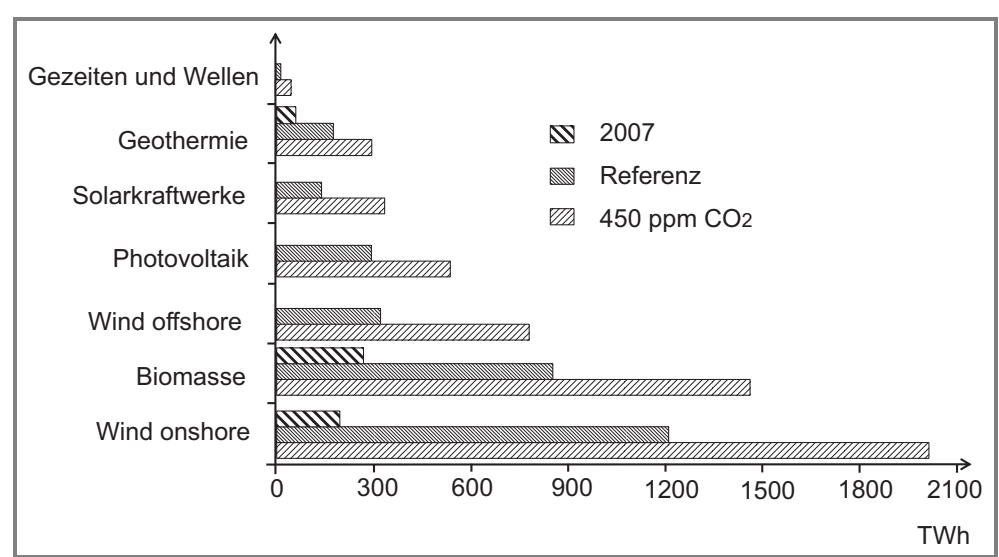

Abbildung 1. Szenarien zur weltweiten Stromerzeugung aus erneuerbaren Energien im Jahr 2030 [5]. Im Szenario „Referenz" steigt der maximale $\mathrm{CO}_{2}$-äquivalent Anteil in der Atmosphäre auf etwa 1000 ppm an. Im Szenario "450 ppm $\mathrm{CO}_{2}$ “ ist ein maximaler $\mathrm{CO}_{2}$-äquivalent Anteil in der Atmosphäre von $450 \mathrm{ppm}$ vorgese- 
[7] bindende Vorgaben formuliert. Bis zum Jahr 2020 sollen gemäß den Zielen der RED 20\% der Energie aus erneuerbaren Energieträgern und $10 \%$ der Kraftstoffe aus Biomasse gewonnen werden.

In den Szenarien stellt die Windenergie bis 2020 den bedeutsamsten erneuerbaren Energieträger zur Stromerzeugung dar, gefolgt von der Biomasse. Bis 2030 wird auch die Solarenergie bei der Stromerzeugung deutlich an Bedeutung gewinnen.

\section{Biomasse und Ersatzbrennstoffe}

Der Begriff Biomasse deckt eine Vielzahl von Brennstoffen ab. Allgemein umfasst dieser Begriff alle nicht-fossilen organischen Pflanzen- und Tiermassen. Aus energetischer Sicht fallen darunter neben holzartige Biomassen auch landwirtschaftliche Produkte und Abfälle wie Stroh, Gülle, Torf und Klärschlamm. In Deutschland zählen Torf und Klärschlamm aus Sicht der Energieerzeugung allerdings nicht zur Biomasse [8]. Torf wird in Deutschland so gut wie nicht zur energetischen Nutzung eingesetzt. Um den europaweiten Umgang mit den festen Biomassebrennstoffen $\mathrm{zu}$ erleichtern, wird aktuell die DIN EN Norm 14961 eingeführt [9], in der Holz, Pellets und feste biogene Reststoffe aus der Landwirtschaft und dem Gartenbau für die energetische Nutzung definiert sind.

Wie bereits angedeutet, werden aufgrund des Kostendrucks Reststoffe und schnell wachsende Biomassen an zunehmend wichtiger. Dazu zählen vor allem Landschaftspflegematerial, Stroh, Miscanthus, Pappeln und weitere neuere Sorten.

Im Rahmen der Reststoffe wird auch Klärschlamm verstärkt an Bedeutung gewinnen. Der Klärschlamm unterliegt der Klärschlamm- und Abfallablagerungsverordnung $[10,11]$, die eine Ausbringung auf landwirtschaftliche Flächen und die Deponierung von Klärschlamm regelt. Die Verschärfung der Vorgaben, insbesondere die Limitierung der organischen Bestandteile (Glühverluste) auf $5 \%$, erfordert in vielen Fällen eine thermische Verwertung. Somit entwickelt sich Klärschlamm zu einem Energieträger, der vor allem im Bereich der Mitverbrennung und Abfallverwertung in Kraftwerken eingesetzt wird.

Die Zusammensetzung der Biomasse ist für deren energetische Nutzung von großer Bedeutung. Vor allem unterscheiden sich der Asche-, Wasser-, Schwefel-, Chlor-, Schwermetall- (z. B. Cd, Zn) und Alkaliengehalt (K, Na, Ca, Mg). Während die Schwermetalle die Nutzung der Asche definieren, beeinflussen die Alkalien vor allem die Ascheerweichungstemperatur. Bei dieser Temperatur zeigen sich erste Anzeichen der Erweichung und des Aufblähens der Asche [12].

Entsprechend dem Chlor- und Alkaligehalt der Biomasse unterscheidet sich auch das Korrosionspotenzial an den Kesselwänden und Wärmeübertragerflächen erheblich. Insbesondere die chlorreichen Brennstoffe wie Stroh sind diesbezüglich kritisch (s. a. Tab. 3).
Der Wassergehalt $(w)$ der Brennstoffe wird definiert als das Verhältnis von Masse des Wassers zu Masse des feuchten Brennstoffs (Gl. (1)) und variiert stark.

$w=\frac{\text { Masse Wasser }}{\text { Masse absolut trockener Brennstoff }+ \text { Masse Wasser }} \cdot 100 \%$

Frisches Holz kann bis $\mathrm{zu}$ 60\% Wasser enthalten. Getrocknet sind Werte von 20-35\% üblich. Holzpellets haben mit 8-10\% den geringsten Wassergehalt. Ebenfalls relativ trocken sind Stroh und Getreide mit etwa $15 \%$ Wasser. Frischer Klärschlamm enthält 75-90\% Wasser und wird zur energetischen Nutzung auf Werte von 8-25\% getrocknet.

Der untere Heizwert (Hu) der Brennstoffe wird vom C-, H-, N-, S-, O- sowie dem Wassergehalt definiert und kann gemäß Gln. (2) und (3) berechnet werden [13]. Dabei wird zuerst mit Gl. (2) der Heizwert eines idealen wasserfreien Brennstoffes berechnet und mit Gl. (3) die Verdampfungswärme des enthaltenen Wassers abgezogen und auf den feuchten Brennstoff umgerechnet.

$$
\begin{aligned}
& \mathrm{Hu}_{\text {wasserfrei }}=\frac{34,835 \cdot \mathrm{C}+93,87 \cdot \mathrm{H}+6,28 \cdot \mathrm{N}+10,465 \cdot \mathrm{S}-10,8 \cdot \mathrm{O}}{100}\left[\frac{\mathrm{MJ}}{\mathrm{kg}_{\text {trocken }}}\right] \\
& \mathrm{Hu} u_{\text {feucht }}=\frac{\mathrm{Hu}_{\text {wasserfei }}(100-w)-2,443 w}{100}\left[\frac{\mathrm{MJ}}{\mathrm{kg}_{\text {feucht }}}\right]
\end{aligned}
$$

Als Alternative zur Biomasse werden vor allem in Deutschland in der Industrie sogenannte Ersatzbrennstoffe eingesetzt. Ersatzbrennstoffe werden aus Gewerbe- und Siedlungsabfällen gewonnen. Die Bestandteile von Ersatzbrennstoffen sind heizwertreiche Polymerfraktionen und biogene Abfälle. Diese weisen im Vergleich zu Holz hohe Asche-, Chlor-, Schwefel- und Stickstoffgehalte auf. Tab. 3 zeigt beispielhafte Zusammensetzungen verschiedener Energieträger.

\section{Potenziale von Biomasse zur energetischen Nutzung}

Die Potenziale der Biomasse werden in technische, wirtschaftliche und nachhaltige Potenziale unterschieden. Die Anforderungen an die Nachhaltigkeit betreffen die $\mathrm{CO}_{2}$-Neutralität und lokale ökologische Aspekte, wie die Versauerung der Böden, die Wassernutzung, den Erhalt der biologischen Vielfalt und den Schutz von ausgewählten Ökosystemen (Gebirge, tropische Regionen). Aber auch sozioökonomische Aspekte, wie die Verfügbarkeit von Nahrungsmittelanbauflächen und die emissionsarme Nutzung von Biomasse sowie die Vermeidung von gesundheitlichen Folgeschäden durch die Nutzung von Biomasse sind von Bedeutung. Die meisten Untersuchungen beschränken sich allerdings mangels Daten auf das technische Potenzial [15], so dass auch hier nur darauf eingegangen werden kann.

Der Großteil der weltweit eingesetzten Biomasse wird zur traditionellen Biomassenutzung in Form von Brennholz für 
Tabelle 3. Zusammensetzung von Energieträgern und Erweichungstemperatur der Aschen.

\begin{tabular}{|c|c|c|c|c|c|c|c|}
\hline & Elemente & Holz & $\begin{array}{l}\text { Hackschnitzel } \\
\text { mit Rinde }\end{array}$ & Stroh & Weizen & Klärschlamm & $\begin{array}{l}\text { Ersatzbrenn- } \\
\text { stoffe }\end{array}$ \\
\hline \multicolumn{8}{|c|}{ in [\%] der Trockenmasse ausgenommen $\mathrm{Cd}$ und $\mathrm{Zn}$ in $\left[\mathrm{mg} \mathrm{kg}^{-1}\right]$} \\
\hline & & {$[14]$} & {$[15]$} & {$[16,17]$} & {$[14]$} & {$\left[3, \mathrm{eA}^{1}\right]$} & {$\left[3, \mathrm{eA}^{1}\right]$} \\
\hline \multirow{6}{*}{ Hauptelemente } & $\mathrm{C}$ & $50,7-53,1$ & $47,1-49,8$ & $43,5-47,5$ & 46,2 & $23-40$ & $38,6-50,7$ \\
\hline & $\mathrm{H}$ & $5,7-6,3$ & $6,1-6,3$ & $5,03-6,04$ & 5,8 & $3,7-4,5$ & $3,0-5,9$ \\
\hline & $\mathrm{N}$ & $0,04-0,2$ & $0,13-0,54$ & $0,3-1,5$ & $0,39-0,79$ & $2-5$ & 1,5 \\
\hline & $\mathrm{O}$ & $40,1-43,5$ & $42,1-46,1$ & 47,7 & $43,2-46,3$ & $16-22$ & 23,0 \\
\hline & $\mathrm{S}$ & $0,006-0,012$ & $0,015-0,045$ & $0,077-0,2$ & $0,05-0,11$ & $0,6-7$ & $0,08-0,25$ \\
\hline & $\mathrm{Cl}$ & $0,007-0,023$ & $0,004-0,006$ & $0,1-1,1$ & $0,05-0,25$ & $0,02-0,6$ & \\
\hline \multirow{2}{*}{$\begin{array}{l}\text { Schwer- } \\
\text { metalle }\end{array}$} & $\mathrm{Cd}\left[\mathrm{mg} \mathrm{kg}^{-1}\right]$ & $0,1-0,4$ & $0,08-0,81$ & 0,04 & $0,01-0,03$ & n.v. ${ }^{2}$ & n.v. \\
\hline & $\mathrm{Zn}\left[\mathrm{mg} \mathrm{kg}^{-1}\right]$ & $4-40$ & $13-100$ & 4,4 & $66-106$ & n.v. & n.v. \\
\hline \multirow{5}{*}{$\begin{array}{l}\text { Pflanzen- } \\
\text { nährstoffe }\end{array}$} & K & $0,015-0,065$ & $0,13-0,35$ & $0,2-1,9$ & $0,69-1,19$ & n.v. & n.v. \\
\hline & $\mathrm{Ca}$ & $0,038-0,092$ & $0,29-0,7$ & $0,2-0,5$ & $0,28-0,52$ & n.v. & n.v. \\
\hline & $\mathrm{P}$ & $0,0005-0,0145$ & $0,05-0,10$ & $0,03-0,2$ & $0,06-0,1$ & n.v. & n.v. \\
\hline & $\mathrm{Mg}$ & $0,007-0,025$ & $0,04-0,08$ & $0,04-0,13$ & $0,09-0,13$ & n.v. & n.v. \\
\hline & Asche & $0,5-1,0$ & $1-2,0$ & $4,0-12,0$ & $3,6-5,3$ & $39-53$ & $15-25$ \\
\hline \multicolumn{8}{|c|}{ Wassergehalt in [\%] der Feuchtmasse und Asche-Erweichungstemperatur in $\left[{ }^{\circ} \mathrm{C}\right][15]$} \\
\hline Wassergehalt & & $8-45$ & $20-60$ & $8-15$ & 15 & $5-55$ & $8-15$ \\
\hline Asche-Erweichur & stemperatur & $1283-1426$ & $<1440$ & $911-1.050$ & $687-730$ & & \\
\hline
\end{tabular}

1) eA: eigene Analyse, 2) n.v.: Werte nicht verfügbar.

einfachste Heizzwecke verwendet [18]. Das Potenzial aller Biomassearten weltweit liegt bei rund 104 EJ oder ca. 20,6\% des Primärenergiebedarfs $[5,15]$. In der EU-27 beträgt das Potenzial der Biomasse rund 7456 PJ oder ca. 10,1-13,3\%. Dieses Potenzial setzt sich zusammen aus holzartiger Biomasse (2771 PJ), Reststoffen wie Restholz, Stroh, Ernterückstände und Klärschlamm (3609 PJ) und Energiepflanzen (mind. 1076 PJ). Ein darin größtenteils noch nicht erschlossenes Potenzial stellen Stroh und Energiepflanzen dar. In Deutschland bietet die Biomasse ein Potenzial von 7,6 - 10,2 \% zur Deckung des Primärenergiebedarfs (Tab. 4).

Eine Steigerung des Biomasseanteils in Deutschland oder der EU über diese Werte hinaus kann nur durch Importe

Tabelle 4. Potenzial zur Deckung des Primärenergiebedarfs (PE) in der EU-27 und in Deutschland $[15,19]$.

\begin{tabular}{lccc}
\hline Ressource & $\begin{array}{c}\text { Beitrag } \\
\text { weltweit [E]] }\end{array}$ & $\begin{array}{c}\text { Beitrag } \\
\text { EU-27 [P]] }\end{array}$ & $\begin{array}{c}\text { Beitrag } \\
\text { Deutschland [P]] }\end{array}$ \\
\hline Waldrestholz & 41,6 & 2771 & 421 \\
Reststoffe & 24,8 & 3609 & 458 \\
Energiepflanzen & 37,4 & $1076-3433$ & $186-561$ \\
Summe & 103,8 & $7456-9.813$ & $1065-1440$ \\
PE-Bedarf & 502,9 & 73600 & 14012 \\
\hline
\end{tabular}

von Biomasse von außerhalb der EU ermöglicht werden. Zum Transport über weitere Strecken eignen sich vor allem feste Biomassen wie Holzpellets sowie leitungsgebundene synthetische Energieträger (Substitute Natural Gas), die aus Biomasse gewonnen werden können.

\section{Die Aufbereitung von Biomasse}

Waldhackgut, pelletierte Reststoffe aus der Sägeindustrie und Reststoffe der Papierindustrie stellen den Hauptanteil der Holzbrennstoffe dar. Aktuell wird vor allem die Herstellung von Holzpellets international stark ausgebaut. Die Pelletierung dient vor allem der Erhöhung der Energiedichte und der Verbesserung der Transporteigenschaften.

Beispiele für geplante Anlagen sind ein Pelletierwerk in den USA mit $750000 \mathrm{ta}^{-1}$ (RWE) und eine Anlage in Russland mit $900000 \mathrm{ta}^{-1}$ (Vyborgskaja Cellulosa). Neben den USA und Russland stehen in Kanada und Schweden weitere, größere Ressourcen an Biomassen zur Herstellung von Holzpellets zur Verfügung. Hauptabsatzmärkte für diese Länder sind Europa und Asien. Somit entwickeln sich Holzpellets zu einem Exportgut, das auch über weitere Strecken transportiert wird.

Weltweit werden derzeit etwa 12 Mio. t Holzpellets pro Jahr produziert, davon rund die Hälfte in der EU. In 
Deutschland wurden in 2010 etwa 1,75 Mio. t erzeugt und 1,2 Mio. $t$ im Land verbraucht [20]. In den USA dürfte der Verbrauch derzeit ähnlich groß sein [21,22]. Österreich verbrauchte zum Vergleich in 2010 etwa 0,66 Mio. t [23]. Bei den derzeitigen Verbräuchen an Biomasse beträgt der energetische Anteil der Pellets an der Biomasse in Deutschland und der EU allerdings nur etwa 2,5\%.

Seit einigen Jahren werden auch thermochemische Verfahren zur Behandlung von lignozellulosehaltiger Biomasse entwickelt und erste Pilotanlagen errichtet. Die Verfahren sind unter den Bezeichnungen Torrefaction (TF) und Hydrothermale Karbonisierung (HTC) bekannt. Mehrere Unternehmen arbeiten derzeit vor allem in den Niederlanden und Deutschland an der Verfahrensentwicklung. Teilweise basieren die Verfahrensentwicklungen auf Entwicklungen und ersten Untersuchungen aus der ersten Hälfte des 20. Jahrhunderts [24-26].

Das Ziel der TF- und HTC-Verfahren ist die Erhöhung des spezifischen Kohlenstoffanteils in der Biomasse durch Verkohlung organischer Bestandteile der Biomasse. Bei der Behandlung kommt es zu einer Verringerung der flüchtigen Bestandteile und einer Reduzierung an Holzmasse.

Für die Torrefizierung wird überwiegend trockene Biomasse bei $200-300^{\circ} \mathrm{C}$ für $15-120 \mathrm{~min}$ bei atmosphärischem Druck unter Sauerstoffausschluss eingesetzt. Bei der Hydrothermalen Karbonisierung erfolgt die Behandlung in Wasser, wodurch sich auch nasse Biomasse sehr gut eignet. Der Prozess läuft für mehrere Stunden unter Sauerstoffabschluss bei Temperaturen bis $\mathrm{zu} 250^{\circ} \mathrm{C}$ und bei Drücken von $10-20$ bar.

Man geht bei den Verfahren von einer langsamen Pyrolyse unter Luftsauerstoffauschluss aus, bei der eine Ligninerweichung, Karbonisierung von Hemizellulose und Zersetzung in flüchtige Bestandteile und Kohlenstoff stattfindet. Es bilden sich dabei Öle, die polymerisieren und verkohlen. Der Verlust an Holzmasse beträgt 10-20\%. Da die Prozesse exotherm sind, fällt Abwärme an, so dass der energetische Wirkungsgrad der Verfahren $90-96 \%$ beträgt [27-30].

Je nach Verfahren bildet sich ein holzkohlenartiges, der Braunkohle sehr ähnliches Produkt aus. Insbesondere beim nassen HTC-Verfahren ist zusätzlich ein entsprechend energieeffizienter Trocknungsprozess erforderlich. Über eine Reduzierung von Chlor, Schwefel und Alkalien aus dem Brennstoff liegen keine belastbaren Aussagen vor. Es wird angenommen, dass die Reduzierung bei der Torrefizierung gering ist [31], während beim HTC-Verfahren aufgrund der wässerigen Phase Abnahmen Dok? von $\mathrm{K}, \mathrm{Cl}$ und $\mathrm{S}$ durch eine Auswaschung möglich sind. In [32] konnte durch eine Pyrolyse von Stroh und eine nachfol- gende Waschung des Restkoks mit Wasser gezeigt werden, dass $70 \%$ des Kaliums, $90 \%$ des Chlors und 36\% des Schwefels aus dem Koks entfernt werden können.

Da TF- und HTC-Biokohlen einen im Vergleich zu Holz erhöhten Heizwert aufweisen und die Porosität zunimmt, sind sowohl eine Pelletierung als auch eine Mahlung der Biokohlen interessant. Dadurch eröffnen sich weitere Möglichkeiten für den Transport und die thermische Nutzung der Biomasse. Tab. 5 zeigt den Vergleich von Holzhackgut, Holzpellets, TF- und HTC-Biokohlen.

\section{Thermische Nutzung von Biomasse}

Die verschiedenen Möglichkeiten der Umwandlung von Biomasse sind in Abb. 2 dargestellt. Die thermische Nutzung von Biomasse basiert auf Verbrennungs- und Vergasungsprozessen. Während die Verbrennung als Stand der Technik angesehen werden kann, ist die thermochemische Umwandlung mittels der Vergasungsprozesse - von wenigen Ausnahmen abgesehen - von einer breiten kommerziellen Umsetzung noch entfernt. Die Ursachen dafür sind meist technische Probleme beim Betrieb der Anlagen.

Die Nutzung von Holzpellets erfolgt in Mitteleuropa und in den USA nahezu ausschließlich in Kleinanlagen $<200 \mathrm{~kW}$ [34-36]. In den skandinavischen Ländern, den Benelux-Staaten und Großbritannien ist an transportgünstigen Standorten, insbesondere an der Küste, die Mitverbrennung von Holzpellets oder ausschließliche Nutzung von Pellets in Großkesselanlagen stärker verbreitet. Dänemark und Großbritannien importieren einen Großteil der Holzpellets auf dem Schiffsweg und nutzen diese zur Mitverbrennung. Die Nutzung erfolgt entweder in Wirbelschichtfeuerungen oder in gemahlener Form mit Kohlestaubbrennern. Bei der Mitverbrennung in Kohlestaubfeuerungen beschränkt sich der Anteil aufgrund der Korrosion, der Ascheablagerungen und der Ascheerweichung meist auf $<20 \%$. Anteile von bis $\mathrm{zu} 50 \%$ an Biomasse werden teilweise angestrebt [37, 38].

Bei den Feuerungskonzepten für Biomasse konzentrieren sich die aktuellen Forschungsarbeiten auf die Entstehung und Reduzierung von Emissionen, die Partikelentstehung

Tabelle 5. Vergleich der Eigenschaften behandelter Biomassen.

\begin{tabular}{lccccc}
\hline $\begin{array}{l}\text { Eigenschaft/ } \\
\text { Biomasse }\end{array}$ & $\begin{array}{c}\text { Hackgut } \\
\text { typische } \\
\text { Werte }\end{array}$ & $\begin{array}{c}\text { Holzpellets } \\
{[28]}\end{array}$ & $\begin{array}{c}\text { TF-Hackgut } \\
{[28]}\end{array}$ & HTC-Pulver & TF-Pellets \\
\hline Wassergehalt $[\% \mathrm{f}]$ & 35 & $7-10$ & 3 & 8 & {$[33]$} \\
Heizwert $\mathrm{Hu}_{\mathrm{feucht}}\left[\mathrm{MJ} \mathrm{kg}^{-1}\right.$ feucht] & $10,5-10,9$ & $15,6-16,2$ & 19,9 & 20 & $19,9-21,6$ \\
Schüttdichte $\left[\mathrm{kg} \mathrm{m}_{\mathrm{f}}^{-3}\right]$ & $226-260$ & $520-650$ & 230 & n.v. & $750-850$ \\
Energiedichte $\left[\mathrm{GJ} \mathrm{m} \mathrm{f}^{-3}\right]$ & $2,45-2,85$ & $7,8-10,5$ & 4,6 & n.v. & $14,9-18,4$ \\
Aschegehalt $\left[\% \mathrm{tr}_{\mathrm{tr}}\right]$ & $1,5-2$ & $0,5-1$ & n.v. & 1,5 & n.v. \\
\hline
\end{tabular}

f: feucht, tr: trocken, n.v.: Werte nicht verfügbar 


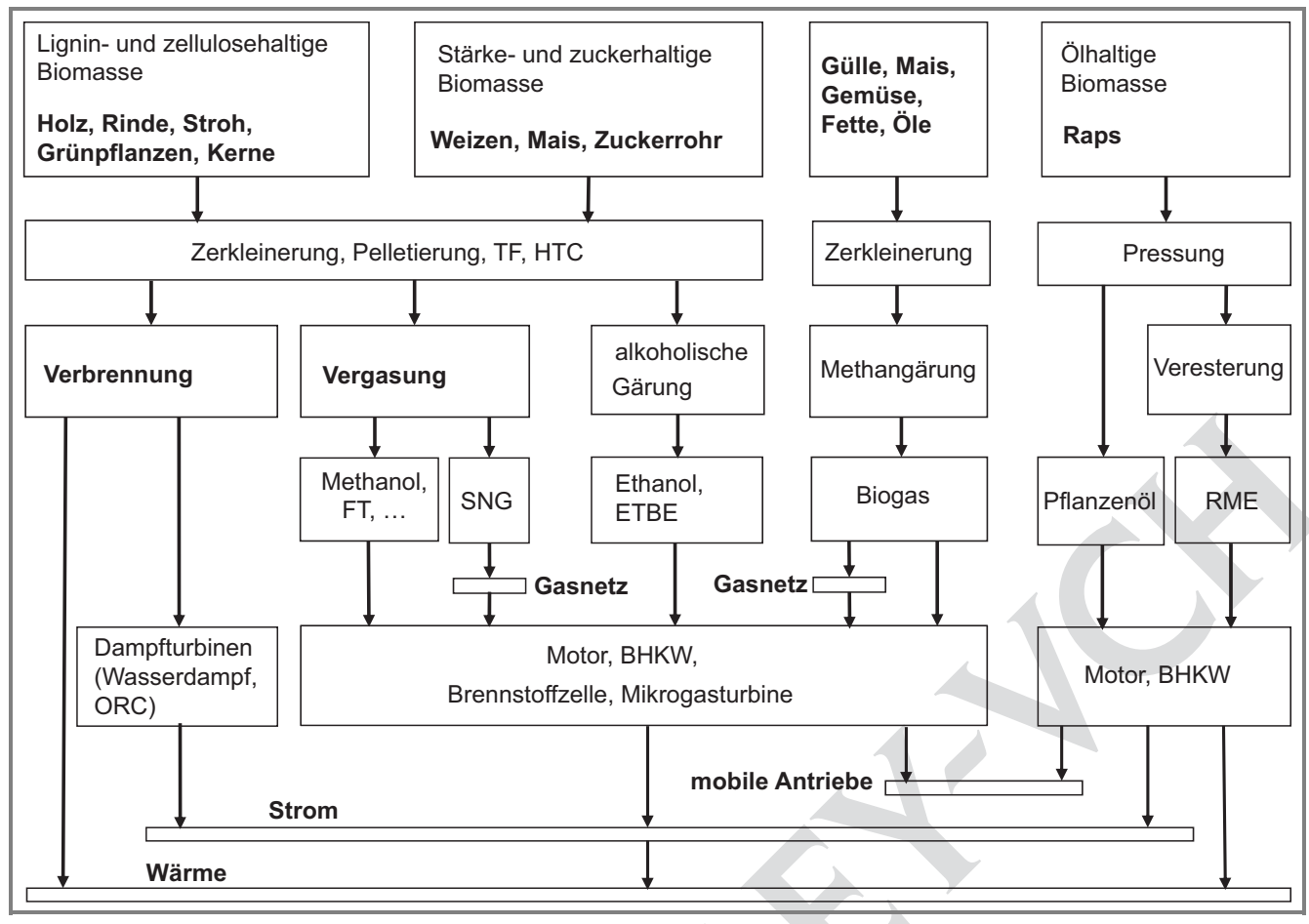

Abbildung 2. Möglichkeiten der energetischen Nutzung von Biomassen. RME: Rapsmethylester, SNG: Substitute Natural Gas, ETBE: Ethyltertiärbutylether, FT: Fischer-Tropsch-Synthese, ORC: Organic Rankine Cycle.

und deren Vermeidung, die Korrosionsvermeidung in Feuerungen und an Dampferzeugern sowie die Nutzungsmöglichkeiten der Rostasche.

Bei der Vergasung stehen Untersuchungen zur Teerentstehung und Reduzierung, die Abscheidung von Partikeln, die Verstromung und die Aufbereitung des Produktgases zu einem Erdgasersatzprodukt (SNG) oder einem flüssigen Energieträger (Fischer-Tropsch-Synthese) im Mittelpunkt.

\subsection{Verbrennung und Feuerungstechnik}

Die thermische Nutzung von Biomasse und Abfallstoffen erfolgt aktuell vorwiegend über Feuerungsanlagen. In den letzten Jahrzehnten wurden die Feuerungskonzepte an die Bedürfnisse der Biomasse besser angepasst, die Effizienz gesteigert und die Emissionen an $\mathrm{CO}$ und Partikeln reduziert. Verschiedene Systeme sind verfügbar und wurden in einer Vielzahl von realisierten Anlagen erprobt. In Abb. 3 sind die Teilschritte der Verbrennung von Biomasse im Feuerraum dargestellt. Die Anforderungen an den Verbrennungsprozess werden bestimmt durch:

- Trocknung und Erwärmung des Brennstoffes

- Pyrolyse und Vergasung der flüchtigen Bestandteile

- Oxidation des verbleibenden Restkoks

An Parametern zur Auslegung und Auswahl des Feuerungssystems stehen die Verbrennungsführung (Luftstufung, Temperatur), die Vermischung (Turbulenz) der Gase mit der Verbrennungsluft und die Ver- weilzeit im Feuerraum zur Verfügung. Voraussetzung für einen guten Ausbrand von Kohlenstoff ist eine ausreichende Verweilzeit bei ausreichender Temperatur und ausreichendem Sauerstoffangebot.

Eine Aufteilung in eine primäre und eine sekundäre Verbrennungszone und eine gestufte Verbrennungsluftzugabe ermöglicht die Beeinflussung der Verbrennungstemperatur. In der primären Verbrennungszone werden unterstöchiometrische Bedingungen mit einem Lambda $\lambda$ von $0,4-0,8$ realisiert. Dadurch wird die Verbrennungstemperatur limitiert und eine Erweichung der Asche vermieden.

Die sogenannte Luftzahl Lambda $\lambda$ stellt nach Gl. (4) das Verhältnis der zugeführten Verbrennungsluftmenge $\left(L_{\text {eff }}\right)$ zur Luftmenge, die theoretisch zur vollständigen Verbrennung des Brennmaterials benötigt wird $\left(L_{\mathrm{min}}\right)$, dar.

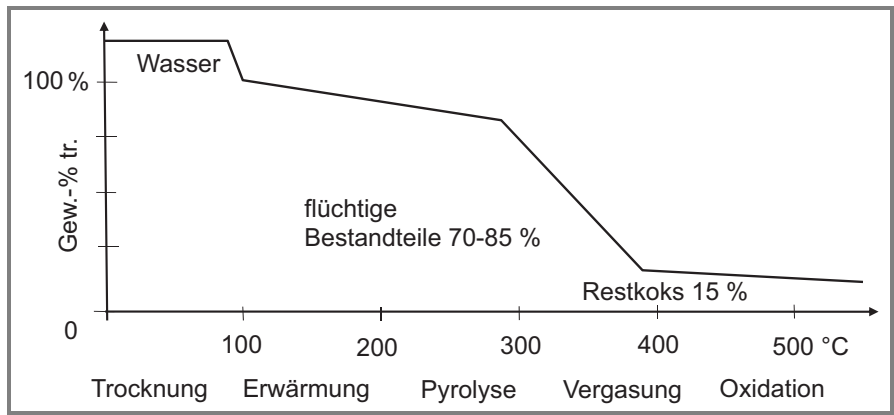

Abbildung 3. Die Abläufe während der Verbrennung von Biomasse in Abhängigkeit der Temperatur am Beispiel von feuchtem Holz [39]. 
$\lambda=\frac{L_{\text {eff }}}{L_{\min }} \quad[-]$

In der sekundären Verbrennungszone wird weiter Verbrennungsluft zugeführt, um bei einem $\lambda$ von 1,3 bis etwa 2,0 eine möglichst vollständige Oxidation von Kohlenmonoxid und Kohlenwasserstoffen $\mathrm{zu}$ gewährleisten. Dabei kann die Temperatur nahe an den Erweichungspunkt der Flugaschepartikel (s. Tab. 3) ansteigen. In der Regel werden Werte $<1050^{\circ} \mathrm{C}$ eingehalten. Als Richtwerte für gute Verbrennungsbedingungen gelten eine Temperatur von mindestens $850^{\circ} \mathrm{C}$ und eine Verweilzeit von $0,5-1,0$ s der Gase in der heißen Verbrennungszone.

Um die Verbrennungstemperatur zu begrenzen, kann der Luftüberschuss erhöht oder feuchterer Brennstoff eingesetzt werden. Der Brennstoffwassergehalt sollte maximal $55-60 \%$ betragen. Ein hohes $\lambda$ in der Kombination mit einem nassen Brennstoff führt allerdings zu einer Absenkung der Verbrennungstemperatur, so dass Emissionen von Kohlenmonoxid stark ansteigen können.

Bei der Erhöhung des Luftüberschusses oder des Wassergehalts sinkt der Wirkungsgrad. Eine alternative Möglichkeit der Temperaturbegrenzung ist eine Rückführung von Abgasen mit Temperaturen von $100-200^{\circ} \mathrm{C}$ nach dem Wärmeübertrager in den Feuerraum (Abgasrezirkulation). Da bei der Abgasrezirkulation der Wirkungsgrad gleich bleibt, ist diese Methode $\mathrm{zu}$ bevorzugen. Allerdings erhöht sich dadurch der technische Aufwand an der Feuerung.

\subsection{Feuerungskonzepte}

Die Auswahl der Feuerungstechnik richtet sich überwiegend nach der Beschaffenheit des Brennstoffs. Entscheidende Parameter sind der Wassergehalt, Aschegehalt, Temperatur der Ascheerweichung, Stückigkeit und der Schadstoffgehalt. In Abb. 4 sind die jeweiligen Einsatzbereiche der Feuerungsarten dargestellt.

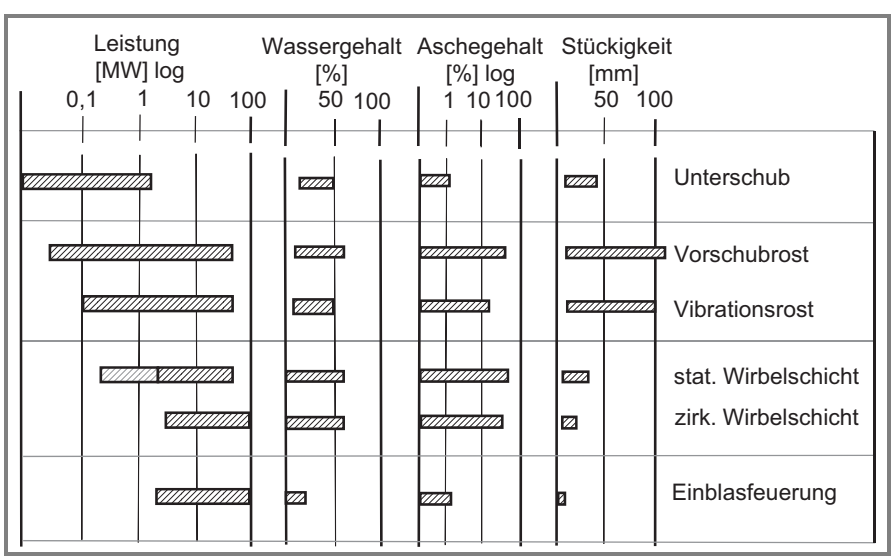

Abbildung 4. Typische Größenordnungen und Betriebsbereiche verschiedener Feuerungsarten. Dargestellt sind die Feuerungsleistung, der Wasserund Aschegehalt und die Stückigkeit des eingesetzten Brennstoffs [40].

\subsubsection{Unterschubfeuerung}

Im kleinen $(10-100 \mathrm{~kW})$ und mittleren $(100-1000 \mathrm{~kW})$ Leistungsbereich werden für die Verfeuerung von Pellets und Hackgut überwiegend Unterschubfeuerungen eingesetzt. Diese eignen sich vor allem für Brennstoffe mit einem Aschegehalt < 1,5\%. Die Brennstoffzuführung limitiert hier in der Regel die Partikelgröße des Brennstoffs, so dass kleinere Anlagen mit Holzpellets und Hackgut mit einer Brennstoff-Stückgröße $<5 \mathrm{~cm}$ betrieben werden können. Der Wassergehalt ist bei Anlagen $<200 \mathrm{~kW}$ meist mit $35 \%$ limitiert.

\subsubsection{Rostfeuerung}

Rostfeuerungen sind in verschiedenen Ausführungen, wie Stufenrost und Vorschubrost, verfügbar. Üblich ist die Bauform ab etwa $500 \mathrm{~kW}$ Leistung, einfache Roste sind aber bereits ab etwa $50 \mathrm{~kW}$ erhältlich. Rostfeuerungen eignen sich auch für Brennstoffe mit einem sehr hohen Aschegehalt bis zu $30 \%$ und einem Wassergehalt bis zu $60 \%$. Für Brennstoffe mit Ascheerweichungstemperaturen $<850^{\circ} \mathrm{C}$ werden die Rostelemente und der Brennraum oft wassergekühlt ausgeführt. Durch die im Vergleich zum Unterschubsystem meist größere Brennstoffmenge im Brennraum verlaufen Lastwechsel träge und ein Ein-Aus-Betrieb ist schwierig. Die Feuerung wird meist im Aus-Betrieb mit einem sogenannten Gluterhalt-Betrieb warmgehalten, um ein automatisches Anfahren zu ermöglichen.

Weitere Bauformen für Festbettfeuerungen sind Vibrationsroste, Flachvorschubroste, Drehtellerroste, Wanderroste, Walzenroste, Brennkegelfeuerungen, Drehrohröfen, Zigarren- und Ballenbrenner [41, 42].

\subsubsection{Wirbelschichtfeuerung}

Wirbelschichtfeuerungen werden ab etwa $10 \mathrm{MW}$ realisiert. Man unterscheidet stationäre, blasenbildende und zirkulierende Wirbelschichten. Unterhalb $70 \mathrm{MW}$ werden aufgrund der Kosten meist stationäre, darüber zirkulierende Wirbelschichten eingesetzt. Die Vorteile der Wirbelschicht sind eine gut realisierbare Luftstufung mit $\lambda$-Werten von 0,4 im Wirbelbett, geringe CO-Emissionen und ein im Vergleich zur Rost- oder Unterschubfeuerung deutlich geringerer Luftüberschuss von $2-3 \% \mathrm{O}_{2}$ im Abgas. Das entspricht einem $\lambda$ von 1,2-1,3.

Eine Einteilung der verschiedenen Feuerungsarten kann beispielsweise nach dem Druckverlust über das Brennstoffbett in Abhängigkeit der Strömungsgeschwindigkeit erfolgen. Entsprechend Abb. 5 ergibt sich für die Festbettfeuerungen bis zum Lockerungspunkt ein nahezu linearer Druckanstieg, während für stationäre Wirbelschichten der Druckverlust relativ konstant bleibt und erst ab dem Austragspunkt weiter ansteigt. Der Druckverlust der Wirbelschicht beträgt etwa 150 mbar (inkl. Düsenboden). Der Wärmeübergang von 


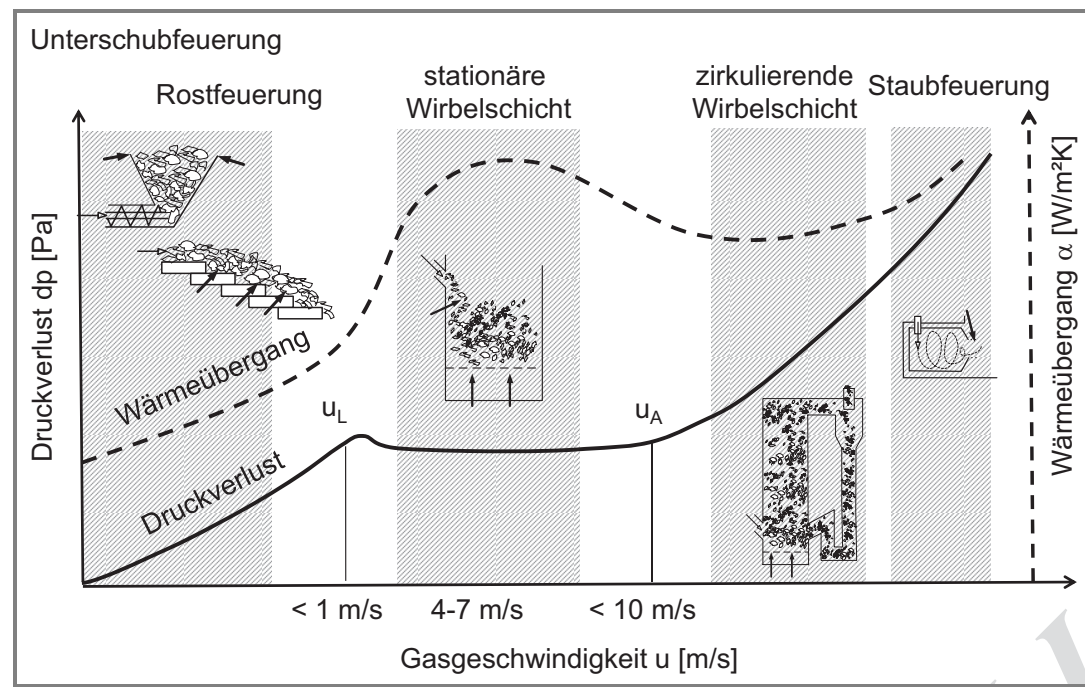

Abbildung 5. Unterschub-, Rost-, stationäre und zirkulierende Wirbelschicht und Staubfeuerung in Abhängigkeit der Gasgeschwindigkeit im Brennraum [15]. $u_{L}$ : Lockerungsgeschwindigkeit der Brennstoffpartikel mit Übergang zur Wirbelschicht, $u_{\mathrm{A}}$ : Austragsgeschwindigkeit mit Übergang zur pneumatischen Förderung der Brennstoffpartikel.

gen arbeiten mit einem $\lambda$ von etwa 1,3 口ok? Rost- und Unterschubfeuerungen werden wegen der vergleichsweise schlechteren Durchmischung von Brennstoff und Verbrennungsluft und dem sehr unterschiedlichen Brennstoffwassergehalt mit $\lambda$-Werten von 1,6-2,0 betrieben.

Der Bereich der CO-Emissionen von Anlagen in der Praxis ist sehr groß. Sehr gut geregelte Anlagen erreichen CO-Werte von $<100 \mathrm{mg} \mathrm{m}^{-3} \mathrm{~N}$, während bei ungeregelten Anlagen wie z. B. alten Scheitholzfeuerungen Werte von über $10000 \mathrm{mg} \mathrm{m}^{-3} \mathrm{~N}$ auftreten können.

$\mathrm{NO}_{\mathrm{x}}$-Emissionen stellen bei den meisten holzartigen Biomassen kein allzu großes Problem dar. Primär bildet sich das $\mathrm{NO}_{\mathrm{x}}$ aus dem im Brennstoff enthaltenen Stickstoff. Bei einer Luftzahl $<0,8$ bildeen sich $\mathrm{HCN}$ und $\mathrm{NH}_{3}$. Bei einer weiteren Sauerstoffzugabe entstehen daraus $\mathrm{NO}_{\mathrm{x}}$ und $\mathrm{N}_{2}$. Die Bildung

Gas zum Wärmübertrager bzw. Wirbelbett zum Wärmeübertrager ist besonders bei der stationären Wirbelschicht mit $100-600 \mathrm{~W} \mathrm{~m}^{-2} \mathrm{~K}^{-1}$ vergleichsweise hoch.

\subsubsection{Verstromung}

Die Verstromung von Biomasse in Kombination mit Verbrennungsprozessen erfolgt nahezu ausschließlich in Turbinenanlagen mit Wasserdampf (Clausis-Rankine-Prozess) oder einem organischen Arbeitsmedium auf Basis des Organic-Rankine-Cycle (ORC). Für Leistungen von $600 \mathrm{~kW}_{\mathrm{el}}$ bis $2 \mathrm{MW}_{\mathrm{el}}$ haben sich einstufige ORC-Turbinen durchgesetzt. Kleinere ORC-Anlagen sind ebenfalls verfügbar, allerdings steigen die spezifischen Kosten stark an. Der erzielbare elektrische Wirkungsgrad bezogen auf den Biomassebrennstoff beträgt etwa $15 \%$ (netto). Die Gesamtwirkungsgrade bei Nutzung der Kondensationswärme zu Heizzwecken beträgt etwa $80 \%$. Durch Einbindung einer Abgaskondensationsanlage können Werte bis $98 \%$ erreicht werden.

Bei Dampfprozessen sind bei größeren Anlagen elektrische Wirkungsgrade bis zu $37 \%$ mit Wirbelschichtfeuerungen und sauberem Hackgut im Luft-Kondensationsbetrieb und ohne Wärmeauskopplung realisierbar. Allerdings betragen die Dampfparameter dabei bereits um $530^{\circ} \mathrm{C}$ und sind damit über der Grenze zur Hochtemperatur-Chlorkorrosion. Mit Gegendruck oder Entnahmekondensationsturbinen können Gesamtwirkungsgrade von 70-85\% realisiert werden.

\subsection{Emissionen bei der Verbrennung von Biomasse}

Der $\lambda$-Wert ist auch von der Art der Feuerung und dem Wassergehalt des Brennstoffs abhängig. Wirbelschichtfeuerun- von Brennstoff- $\mathrm{NO}_{\mathrm{x}}$ ist bei einem Lambda um etwa 1,5 am größten [39]. Da die Verbrennungstemperatur meist unterhalb von $1200^{\circ} \mathrm{C}$ liegt, erfolgt kaum eine Bildung von thermischem oder promptem $\mathrm{NO}_{\mathrm{x}}$. Bei Stroh und trockenen Reststoffen, wie z. B. Altholz, Spanplatten und getrocknetem Klärschlamm ist eine $\mathrm{NO}_{\mathrm{x}}$-Bildung allerdings nennenswert. Ein guter Kompromiss zwischen geringen CO-Emissionen, der Verbrennungstemperatur und geringen $\mathrm{NO}_{\mathrm{x}}$ Emissionen ist daher schwierig [3]. Die Umsetzung von im Brennstoff gebundenem Stickstoff zu molekularen Stickstoff $\left(\mathrm{N}_{2}\right)$ erfolgt vor allem unter reduzierten Bedingungen. Eine Begrenzung von $\mathrm{NO}_{\mathrm{x}}$-Emissionen ist daher durch eine gezielte Verbrennungsluftstufung oder einer Abgasrezirkulation möglich.

Die Partikelemissionen aus Biomassefeuerungen besteht $\mathrm{zu}>90 \%$ aus Feinstäuben mit einer Partikelgröße $<10 \mu \mathrm{m}$. Das Maximum der Partikelanzahlverteilung liegt zwischen 0,03 und 0,1 $\mu \mathrm{m}$ [41]. Da die Staubemissionen sehr kritisch gesehen werden, sind abhängig vom Brennstoff $\mathrm{ab}$ 2014 für Kleinfeuerungen < 1 MW die Staubemissionen mit $20 \mathrm{mg} \mathrm{m}^{-3} \mathrm{~N}$ limitiert [43].

In Tab. 6 sind übliche Emissionen und Richtwerte für die Grenzwerte bei der Verwendung von Biomasse und Reststoffen dargestellt. In Deutschland gelten bei $\geq 1 \mathrm{MW}$ Feuerungswärmeleistung bei der Verwendung von Biomasse, Altholz [44] und Torf die 4. BImSchV [45] und die TA-Luft [46]. Für Anlagen mit einer Stromerzeugung und $\geq 50 \mathrm{MW}$ Feuerungswärmeleistung gilt die 13. BImSchV [47]. Für Klärschlamm, Ersatzbrennstoffe, Abfall und schwer belastetes Altholz (Altholzklassen AIII, AIV nach [44]) gelten verschärfte Emissionsgrenzwerte nach der 17. BImSchV [48]. 
Tabelle 6. missionsgrenzwerte bei der Verfeuerung von Biomasse. Abhängig von der Verordnung gelten unterschiedliche Mittelwerte und ein unterschiedlicher Bezugssauerstoff.

\begin{tabular}{|c|c|c|c|c|c|}
\hline & $\begin{array}{l}\text { Wertebereich } \\
\text { ohne sekundäre } \\
\text { Abgasreinigung }\end{array}$ & $\begin{array}{l}\geq 4 \mathrm{~kW}^{\text {bis }} \\
<1 \mathrm{MW}^{1} \\
1 . \mathrm{BImSchV}\end{array}$ & $\begin{array}{l}\geq 1 \mathrm{MW} \\
\text { 4. BImSchV \& } \\
\text { TA-Luft }\end{array}$ & $\begin{array}{l}>50 \mathrm{MW} \text { zur } \\
\text { Stromerzeugung, } \\
\text { 13. BImSchV }\end{array}$ & $\begin{array}{l}\geq 1 \mathrm{MW} \\
\text { 17. BImSchV }\end{array}$ \\
\hline $\mathrm{CO}\left[\mathrm{mg} / \mathrm{m}_{\mathrm{N}}{ }^{-3}\right]$ & $100-2000$ & $300-1000^{2,3}$ & $150^{5}$ & 150 & 50 \\
\hline $\mathrm{NO}_{\mathrm{x}}\left[\mathrm{mg} / \mathrm{m}_{\mathrm{N}}{ }^{-3}\right]$ & $100-800$ & kein Wert & $250-500^{5}$ & $250-400^{5}$ & 200 \\
\hline Staub $\left[\mathrm{mg} / \mathrm{m}_{\mathrm{N}}{ }^{-3}\right]$ & $60-1000$ & $20-100^{2,4}$ & $20-100^{5}$ & 20 & 10 \\
\hline
\end{tabular}

1) Ausgenommen sind Anlagen für Stroh, Getreide und ähnliche Pflanzen. Für diese Brennstoffe gilt ab $>100 \mathrm{~kW}$ die 4. BimSchV. 2) Je nach Art der Biomasse, Jahr der Inbetriebnahme und Leistung. 3) Ab 2014 gilt für $\leq 500 \mathrm{~kW}$ ein Grenzwert von $400 \mathrm{mg} \mathrm{m}_{\mathrm{N}}{ }^{-3} \mathrm{CO}$ und $>500 \mathrm{~kW}$ ein Grenzwert von $300 \mathrm{mg} \mathrm{m}_{\mathrm{N}}{ }^{-3}$ CO. Ausgenommen sind Feuerungen $>100 \mathrm{~kW}$ für Stroh, Getreide und ähnliche Pflanzen. Für diese Brennstoffe gilt ein Grenzwert von $1000 \mathrm{mg} \mathrm{m}_{\mathrm{N}}{ }^{-3}$. 4) Ab 2014 gelten für alle Biomassen $20 \mathrm{mg} \mathrm{m}_{\mathrm{N}}{ }^{-3}$ Staub mit Ausnahme von Scheitholz. 5) Je nach Leistung und Brennstoff.

\subsection{Emissionsminderung bei Biomassefeuerungen}

Im Fokus der Emissionsminderung stehen bei der Verwendung von unbelasteten Biomassen derzeit die Partikelemissionen. Diese werden mit Zyklonen, Elektrofiltern und Gewebefiltern abgeschieden. Zyklone sind zur Abscheidung von Partikeln $<1 \mu \mathrm{m}$ allerdings nicht ausreichend. Meistens werden zusätzlich oder anstelle des Zyklons Elektrofilter eingesetzt. Elektrofilter sind mittlerweile in verschiedenen Bauformen auch für Kleinanlagen ab $10 \mathrm{~kW}$ Leistung erhältlich [49]. Elektrofilter für Anlagen $<100 \mathrm{~kW}$ scheiden Partikel mit einer Effizienz von 60-80\% ab. Wie in Abb. 6 dargestellt, erreichen größere Anlagen Abscheidegrade bis etwa $98 \%$. Bei kleineren wärmegeführten Anlagen sind allerdings ein laufender Ein-Aus-Betrieb sowie der Teillastbetrieb der Feuerung problematisch für den Betrieb des Elektrofilters.

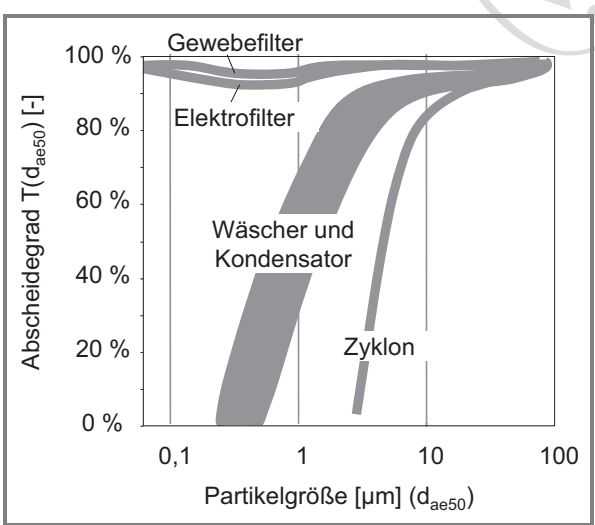

Abbildung 6. Abscheidung von Partikeln mit verschiedenen Technologien [50]. $T\left(d_{\mathrm{ae} 50}\right)$ : Abscheidegrad (Trenngrad) der jeweiligen Partikelfraktion, $d_{\text {ae50 }}$ : aerodynamische Trenndurchmesser mit einem Impaktionswirkungsgrad von $50 \%$ in der Messeinrichtung.

Verstärkt liegt dieser Betrieb bei überdimensionierten Kesselanlagen vor. Da dabei die Abgastemperatur meist unter $100^{\circ} \mathrm{C}$ liegt, können in den Filteranlagen aufgrund von Wasser- und Teerkondensation Betriebsstörungen auftreten. Eine teilweise Abscheidung an Partikeln erfolgt auch mit Abgaskondensationsanlagen. Der erreichbare Abscheidegrad für Partikel beträgt bei Kondensationsanlagen $<50 \%$.

Belastete Brennstoffe verursachen Cl- und S-Emissionen. Zur Abscheidung dieser gasförmigen Schadstoffe wird meistens vor einem Gewebefilter ein Flugstromverfahren mit einem staubförmigen Adsorbens auf Calciumbasis oder Natriumbicarbonat eingesetzt. Dabei wird das Adsorbens in den Abgasstrom als Pulver eingedüst und nach einer Verweilzeit von $1-2 \mathrm{~s}$ am Filter abgeschieden. Flugstrom-Gasreinigungsverfahren in Kombination mit Elektrofiltern sind ebenfalls möglich, besser geeignet sind hier jedoch Gewebefilter. $\mathrm{NO}_{\mathrm{x}}$-Emissionen werden, falls erforderlich, meist mit einem selektiven nicht-katalytischem Verfahren (SNCR) auf Ammoniak-Basis reduziert.

Nachteilig wirken sich der Ein-Aus- und der Teillastbetrieb, wie sie bei Kleinanlagen und monovalenten Einkesselanlagen zur reinen Wärmeversorgung häufig vorkommen, auch auf die Emissionen und den Jahresnutzungsgrad aus. Der Jahresnutzungsgrad beträgt bei diesen Feuerungen meist $<85 \%$. Da die an Wärmeerzeuger angeschlossenen Nahwärmenetze Verluste von $12-30 \%$ aufweisen, beträgt der Nutzungsgrad der gesamten Anlage bei einer reinen Wärmeversorgung meist $<70 \%$. Verbesserungen können ein Pufferspeicher-Management, eine kleiner dimensionierte Feuerungsanlage (bivalente Anlagentechnik) und eine Abgaskondensation bringen [51, 52].

\subsection{Effizienzsteigerung durch eine Abgaskondensation}

Anlagen ab etwa $1 \mathrm{MW}$ Feuerungsleistung können Brennstoffe mit einem Wassergehalt bis zu 60\% verarbeiten. Allerdings sinkt bei einem hohen Wassergehalt im Brennstoff der Wirkungsgrad. Bei sehr feuchten Brennstoffen empfiehlt sich daher die Einbindung einer Abgaskondensation, die einen Teil der Verdampfungswärme bei der Wasserdampf-Kondensationstemperatur des Abgases zurückgewinnt. Die Kondensationstemperatur liegt abhängig vom Wassergehalt und dem Luftüberschuss zwischen 45 und $65^{\circ} \mathrm{C}$. Abb. 7 zeigt den erreichbaren Kesselwirkungsgrad bei Einbindung einer Rauchgaskondensation. Zur Nutzung der Kondensationswärme ist allerdings ein Wärmebedarf bei $<55^{\circ} \mathrm{C}$ erforderlich. Abgaskondensationsanlagen sind für Anlagen ab $10 \mathrm{~kW}$ Leistung bis zur Großanlage verfügbar [41]. 


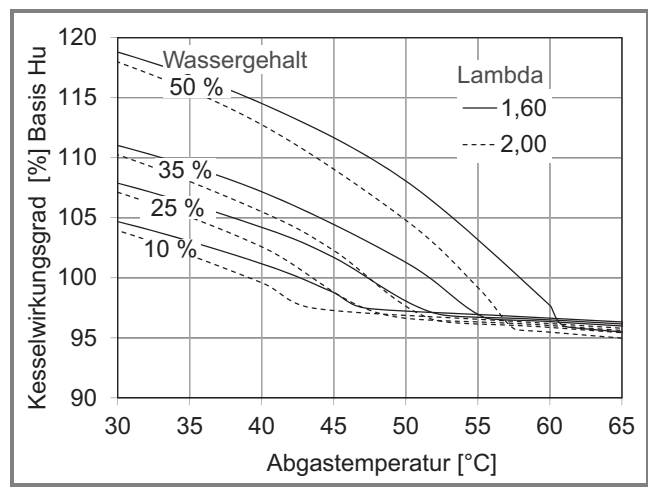

Abbildung 7. Kesselwirkungsgrad mit Rauchgaskondensation bei Verwendung von Hackgut [41]. Basis der Wirkungsgradbeurteilung ist der untere Heizwert (Hu).

\section{Vergasung}

Während die Verbrennung von Biomasse in allen Leistungsklassen Standard ist, wird die Vergasung von Biomasse bisher kaum nennenswert angewandt. Die Vorteile der Vergasung sind eine größere Möglichkeit bei der Nutzung des erzeugten Gasgemischs und ein hoher Verstromungswirkungsgrad bereits bei kleinen Leistungen.

Mit der thermochemischen Umsetzung von festen kohlenstoffhaltigen Energieträgern kann ein brennbarer gasförmiger Energieträger erzeugt werden, der je nach Verfahren aus unterschiedlichen Anteilen von $\mathrm{CO}, \mathrm{H}_{2}, \mathrm{CH}_{4}, \mathrm{CO}_{2}, \mathrm{~N}_{2}$ und $\mathrm{H}_{2} \mathrm{O}$ besteht (s. a. Tab. 8). Zur Vergasung wird ein Oxidationsmittel als Vergasungsmedium (Luft, Sauerstoff oder Wasserdampf) zugegeben. Das Gasgemisch kann in Kraftmaschinen oder zur Herstellung chemischer Grundstoffe verwendet werden.

Die Vergasungsverfahren unterscheiden sich in Ihrer Bauart, dem Oxidationsmittel, der Wärmezufuhr zur Deckung der endothermen Teilreaktionen und dem Betriebsdruck. In der Bauform unterscheidet man:

- Festbettvergaser

- Wirbelschichtvergaser

- Flugstromvergaser

- Sonderbauformen wie Drehrohrvergaser, Rostvergaser, Schneckenvergaser

Am weitesten verbreitet sind Festbettvergaser und Wirbelschichtvergaser. Während die Festbettvergaser aufgrund des
Festbettes in der Leistung nach oben mit einigen MW begrenzt sind, können Wirbelschichtvergaser auch für Leistungen bis $100 \mathrm{MW}$ Brennstoffleistung und mehr eingesetzt werden. Der Flugstromvergaser wird bisher üblicherweise nur für gemahlene Kohle und für Leistungen > $100 \mathrm{MW}$ eingesetzt. Eine Nutzung von Biomasse in Flugstromvergasern gibt es bisher nicht [53]. $\square$ Verweise Abb. 8 \& 9 fehlen

Die Abläufe im Vergaser unterteilen sich in die in Tab. 7 aufgeführten Teilreaktionen. Ein Hauptproblem bei der Vergasung im Festbett und in der Wirbelschicht stellen die bei der Vergasung entstehenden Aromaten dar. Aromate wie Benzol, Toluol und Naphthalin kondensieren bei Abkühlung unter den jeweiligen Taupunkt als feste, flüssige oder hochviskose Produkte. Diese Kondensations- bzw. Sublimationsprodukte werden als Teere bezeichnet. Die Teerbildung beginnt mit der Pyrolyse bei etwa $400^{\circ} \mathrm{C}$, nimmt mit steigender Temperatur zu und erreicht bei Temperaturen zwischen 750 und $900^{\circ} \mathrm{C}$ ein Maximum. Bei noch höheren Temperaturen laufen sekundäre Reaktionen ab, bei denen gebildete Teere wieder abgebaut (gecrackt) werden.

Tabelle 7. Ablauf der Vergasung.

\begin{tabular}{|c|c|}
\hline Prozessstufe $\mathbf{D}$ check & Wirkung $\mathbf{n}$ check \\
\hline Trocknung des Brennstoffes & $\begin{array}{l}\text { Erwärmung und Verdampfung } \\
\text { von Wasser }\end{array}$ \\
\hline Pyrolyse ohne Luftzufuhr & $\begin{array}{l}\text { Freisetzung der flüchtigen } \\
\text { Bestandteile }\end{array}$ \\
\hline Oxidationszone & $\begin{array}{l}\text { Teiloxidation des Restkoks mit } \\
\text { Sauerstoff }\end{array}$ \\
\hline $\begin{array}{l}\text { Vergasung } \\
\text { (Reduktionszone) }\end{array}$ & $\begin{array}{l}\text { Heterogene Teiloxidation des } \\
\text { Restkoks durch Reduktion des } \\
\text { Vergasungsmediums }\end{array}$ \\
\hline $\begin{array}{l}\text { Gasphasenreaktionen } \\
\text { (Reduktion) }\end{array}$ & $\begin{array}{l}\text { Homogene Gasphasenreaktionen } \\
\text { der Flüchtigen }\end{array}$ \\
\hline
\end{tabular}

In realisierten Gleichstrom-Vergasungsanlagen sind Teerwerte von $50-500 \mathrm{mg} \mathrm{m}^{-3}{ }_{\mathrm{N}}$ üblich. Bei den Gegenstromvergasern liegt der Wert bei über $1000 \mathrm{mg} \mathrm{m}^{-3}$. Die Teerkonzentration bei Wirbelschichtvergasern beträgt weit über $1000 \mathrm{mg} \mathrm{m}^{-3}{ }_{\mathrm{N}}$. In der Regel werden mit Wirbelschichtvergasern Teerwerte zwischen 5000 und $10000 \mathrm{mg} \mathrm{m}^{-3}{ }_{\mathrm{N}}$ erreicht.

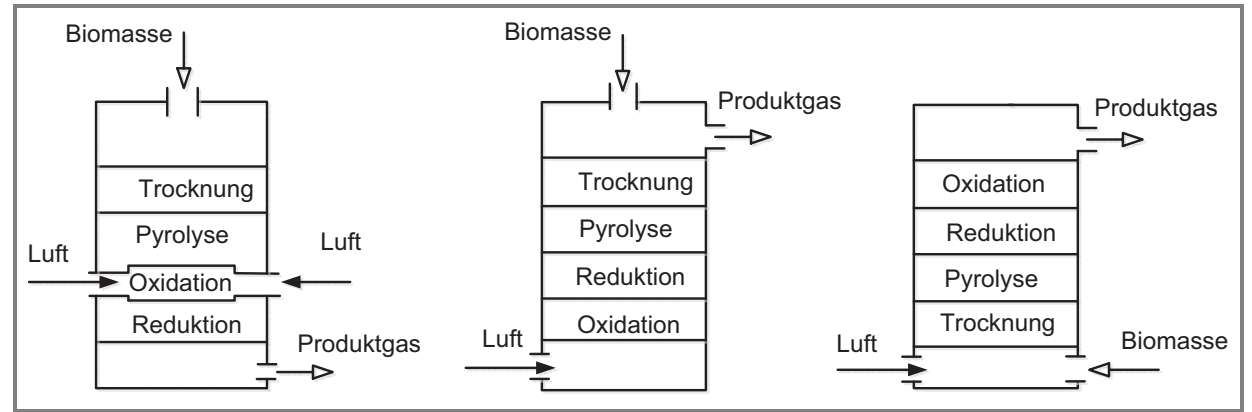

Abbildung 8. Bauformen von Festbett-Vergasern, links: Gleichstrom-absteigend (downdraft), Mitte: Gegenstrom-aufsteigend (updraft), rechts: Gleichstromaufsteigend (updraft) [3]. 


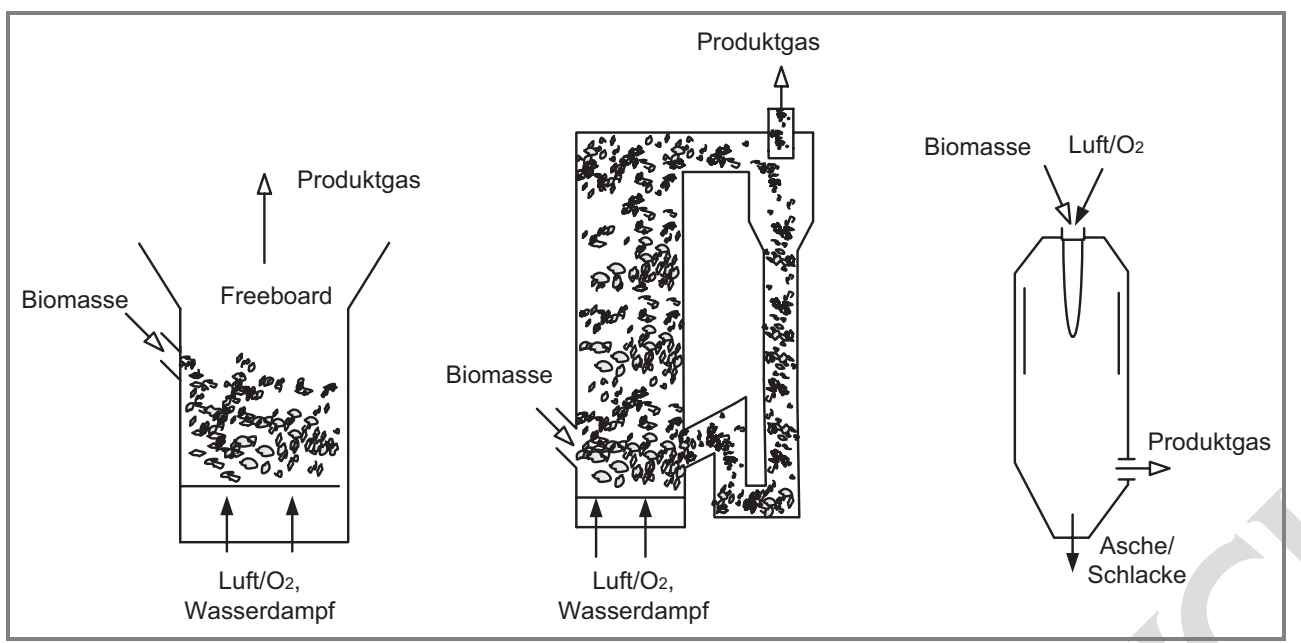

Abbildung 9. Wirbelschichtund Flugstromvergaser, links: stationäre Wirbelschicht, Mitte: zirkulierende Wirbelschicht, rechts: Flugstromvergaser.
In Wirbelschichtvergasern können die Teerkonzentrationen durch die Verwendung eines katalytisch wirkenden Bettmaterials wie Olivin reduziert werden. Bei Flugstromvergasern liegt die Temperatur zwischen 1000 und $1450^{\circ} \mathrm{C}$, so dass die Teerkonzentration abhängig von der Vergasungstemperatur nahezu null beträgt.

Die Reinigung des Produktgases erfolgt je nach Zielsetzung mittels einer Heißgasreinigung mit Keramik- oder Metallfiltern. Zur Reduzierung der Teere können Katalysatoren bei $>850^{\circ} \mathrm{C}$ eingesetzt werden. Alternativ kann abgekühltes Produktgas mit Gewebefiltern und in Wäschern mit Wasser-, Rapsöl- oder Biodiesel gereinigt werden.

Die Nutzung des Produktgasgemischs ist vielfältig. Mit der Vergasung können auch bei kleinen Leistungsbereichen $\left(<500 \mathrm{~kW}_{\mathrm{el}}\right)$ Stromwirkungsgrade bis $27 \%$ (netto, Eigenstromverbrauch berücksichtigt) erreicht werden. Überwiegend werden dazu Gas-Otto-Motoren oder Zündstrahlmotoren eingesetzt. Diese können atmosphärisch oder mit geringem Gasüberdruck betrieben werden. Bei der Nutzung in Gasturbinen ist ein druckaufgeladenes Gas erforderlich. Für Mikrogasturbinen ist ein Druck von 3-5 bar erforderlich [54].

Mit Wirbelschichtvergasern wurde bisher ein Druck von 20 bar $[53,55,56]$ erreicht. In Verbindung mit Flugstromverfahren sind Drücke bis etwa 40 bar realisiert worden. Bei größeren Anlagen kann das Produktgasgemisch somit in GuD-Anlagen (Gas- und Dampf-Kombikraftwerk $\mathbf{D}_{\text {check }}$ ) verstromt werden. Allerdings wird ein durchaus nennenswerter Strombedarf in der Größenordnung von 10-12\% für den Betrieb des Vergasers - Verdichtung des Produktgases (3-5\% der Stromerzeugung), Mahlung und Sauerstoffbereitstellung (5-7\% der Stromerzeugung) - benötigt [3]. In Kombination mit GuD-Anlagen können daher Stromwirkungsgrade von 32-40\% (netto) erreicht werden.

Mit Solid-Oxide- oder Molten-Carbonate-Brennstoffzellen und Kombination von Verstromungsanlagen sind Wirkungsgrade von 42-60\% möglich [54, 57]. Aus Kostengründen werden Brennstoffzellen jedoch bisher kaum verwendet.
Zur Nutzung von Biomasse in Flugstromvergasern werden zweistufige Konzepte konzipiert, bestehend aus einem Festbett-Pyrolysereaktor und einem nachgeschaltetem Flugstromvergaser mit Leistungen von mehreren $100 \mathrm{MW}$. Dabei werden in der ersten Stufe Pyrolyseöl oder -gas und der Pyrolysekoks hergestellt. Diese Produkte werden, wie in Abb. 10 dargestellt, in der zweiten Stufe mit Luft oder Sauerstoff im Flugstromreaktor zu einem Produktgas umgesetzt $[58,59]$.

Aufgrund der im Vergleich zu Wirbelschichten relativ einfachen Bauform von Flugstromvergasern können diese grundsätzlich auch bei kleineren Leistungen von wenigen $10 \mathrm{MW}$ für dezentrale Anlagen verwendet werden. Die karbonisierte Biomasse ist zur Aufmahlung von Partikelgrößen $<200 \mu \mathrm{m}$ gut geeignet und kann daher als Energieträger dienen. Der elektrische Energiebedarf für die Torrefizierung und die Aufmahlung beträgt etwa $18 \mathrm{~kW}_{\mathrm{el}} \mathrm{MW}^{-1} \mathrm{Hu}[53,60]$. Um die Verunreinigung und Verdünnung des Gases durch Stickstoff zu vermeiden, wird beim Flugstromreaktor Sauerstoff als Oxidationsmittel verwendet. Dieser wird mit Druckwechsel-Adsorptionsanlagen (PSA) oder kryogenen Luftzerlegungsanlagen hergestellt.

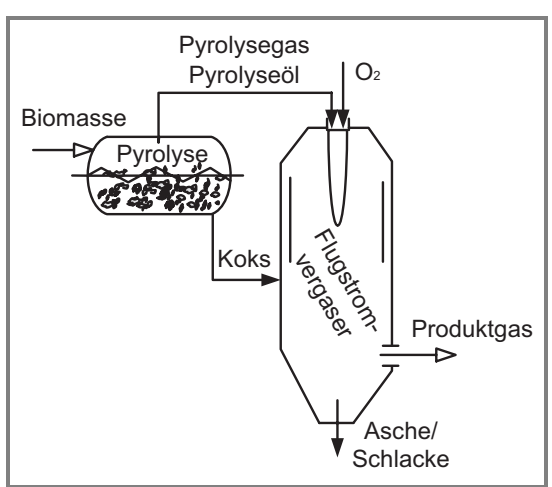

Abbildung 10. Zweistufiger Vergaser mit Pyrolyse und Flugstromvergasung [59]. 
Zur Herstellung eines Gases, das eine Aufbereitung zu einem Erdgasersatz (SNG: Substitute Natural Gas) oder flüssigen Kraftstoff (BtL: Biomass to Liquid) ermöglicht, sind Vergaser geeignet, die mit einem externen Wärmeeintrag betrieben werden oder möglichst reinen Sauerstoff und/oder Wasserdampf als Vergasungsmedium verwenden. Beispiele realisierter allothermer Vergaserkonzepte sind in Abb. 11 dargestellt.

Ähnlich dem Konzept des sogenannten Battelle-Vergasers (Vermont/USA) [61], bei dem Quarzsand als Wärmeträgermedium eingesetzt wird, erfolgte die Entwicklung des intern zirkulierenden Wirbelschichtvergasers der TU Wien (FICFB, Güssing/Österreich) [62]. Nach dem Konzept des in Güssing realisierten Vergasers konnten mittlerweile in Österreich und Deutschland mehrere Anlagen gebaut werden. Mit dem Verfahren sind Leistungen bis etwa $100 \mathrm{MW}_{\text {th }}$ möglich.

Für Leistungen bis zu einigen wenigen $\mathrm{MW}_{\text {th }}$ eignet sich das Heat-Pipe-Reformer-Verfahren [54], das mittlerweile ebenfalls mehrfach als Pilotanlage realisiert wurde. Beim HPR-Verfahren wird die Energie für die Vergasung über Natrium-gefüllte Wärmerohre übertragen. Die Heat-Pipes verbinden eine getrennte Wirbelschichtfeuerung und eine Wirbelschichtvergasung/Reformierung.

Noch in Entwicklung befinden sich das Absorption Enhanced Reforming-Verfahren [63] (AER-Verfahren), bei dem $\mathrm{CaO}$ zur $\mathrm{CO}_{2}$-Abscheidung aus dem Vergaser genutzt wird (Abb. 12). Dabei bildet sich $\mathrm{CaCO}_{3}$, das in der Verbrennungswirbelschicht unter Freisetzung von $\mathrm{CO}_{2} \mathrm{zu} \mathrm{CaO}$ regeneriert wird. Ziel ist ein besonders $\mathrm{H}_{2}$-reiches Produktgas mit einem Anteil von $80 \%$ Wasserstoff. Das Verfahren wird auch als Carbonate Looping bezeichnet.

Die Zusammensetzung des Produktgases ist je nach Vergasungsverfahren sehr unterschiedlich. Einen Überblick zur Zusammensetzung der Produktgasgemische bei verschiedenen Vergasungsverfahren gibt Tab. 8.

Eine weitere Entwicklung eines allothermen Vergasers, der derzeit im Demo-Maßstab existiert, ist das Konzept des sogenannten Milena-Vergasers $[64,65]$. Bei diesem Verfahren ist der Vergaser innerhalb einer Verbrennungswirbel- schicht angeordnet. Der Wärmeintrag erfolgt somit einerseits durch umlaufendes Bettmaterial und andererseits durch das rund um den Vergaser fluidisierte Wirbelbett.

Noch etwas weiter entfernt von der Anwendung sind die sogenannten Chemical-Looping-Verfahren, bei denen durch Nutzung von Metalloxiden ein Wassergas-Shift durchgeführt wird und Wasserstoff hergestellt wird. Dieses Verfahren koppelt an einen Vergaser an und benötigt eine Reduktions- und eine Oxidationszone. Die ablaufenden Reaktionen sind nach [66]:

Reduktion: $\mathrm{C}_{\mathrm{x}} \mathrm{H}_{\mathrm{y}} \mathrm{O}+z \mathrm{Fe}_{2} \mathrm{O}_{3} \rightarrow x \mathrm{CO}_{2}+\gamma / 2 \mathrm{H}_{2} \mathrm{O}+2 z \mathrm{Fe}$ Oxidation:

$3 \mathrm{Fe}+4 \mathrm{H}_{2} \mathrm{O} \rightarrow \mathrm{Fe}_{3} \mathrm{O}_{4}+4 \mathrm{H}_{2}$

$3 \mathrm{FeO}+\mathrm{H}_{2} \mathrm{O} \rightarrow \mathrm{Fe}_{3} \mathrm{O}_{4}+\mathrm{H}_{2}$

$\mathrm{Fe}_{3} \mathrm{O}_{4}+\mathrm{CO} \rightarrow \mathrm{CO}_{2}+3 \mathrm{FeO}$

$\mathrm{Fe}_{3} \mathrm{O}_{4}+\mathrm{H}_{2} \rightarrow \mathrm{H}_{2} \mathrm{O}+3 \mathrm{FeO}$

$4 \mathrm{Fe}_{3} \mathrm{O}_{4}+\mathrm{O}_{2} \rightarrow 6 \mathrm{Fe}_{2} \mathrm{O}_{3}$

\section{Zusammenfassung}

Da für die vorhandenen lignozellulosehaltigen Biomassen die Nachfrage wächst, werden deren Preise steigen und die Bedeutung von kostengünstigen biogenen Reststoffen zunehmen. Um den Bedarf zu decken, wird davon ausgegangen, dass der Import von Biomasse und Energiepflanzen an Bedeutung gewinnt.

Zur Erhöhung der Transportfähigkeit der Biomasse werden neben der Pelletierung weitere Verfahren zur Biomasseaufbereitung entwickelt. Beispiele sind die Torrefizierung und die Hydrothermale Karbonisierung. Daher wird erwartet, dass die Standortwahl für größere Biomasse-Heizkraftwerke sich verstärkt an kosteneffizienten Versorgungswegen wie Flüssen und Küsten orientiert.

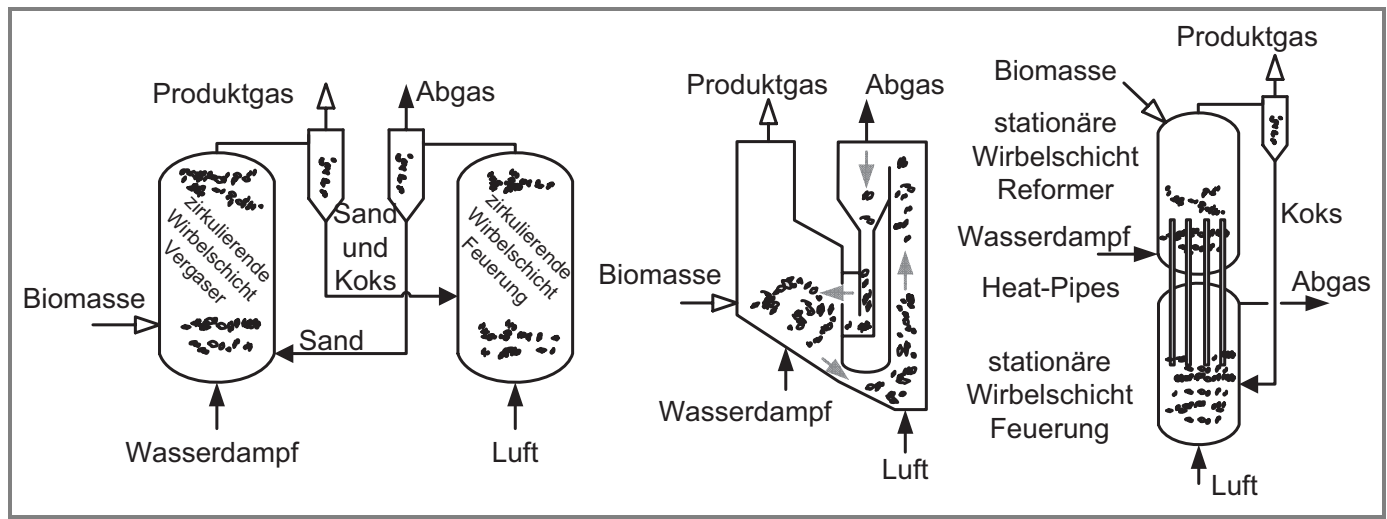

Abbildung 11. Allotherme Vergaserkonzepte, links: Battelle-Vergaser, Mitte: intern zirkulierenden Wirbelschichtvergaser (FICFB), rechts; Heat-Pipe-Reformer (HPR). 


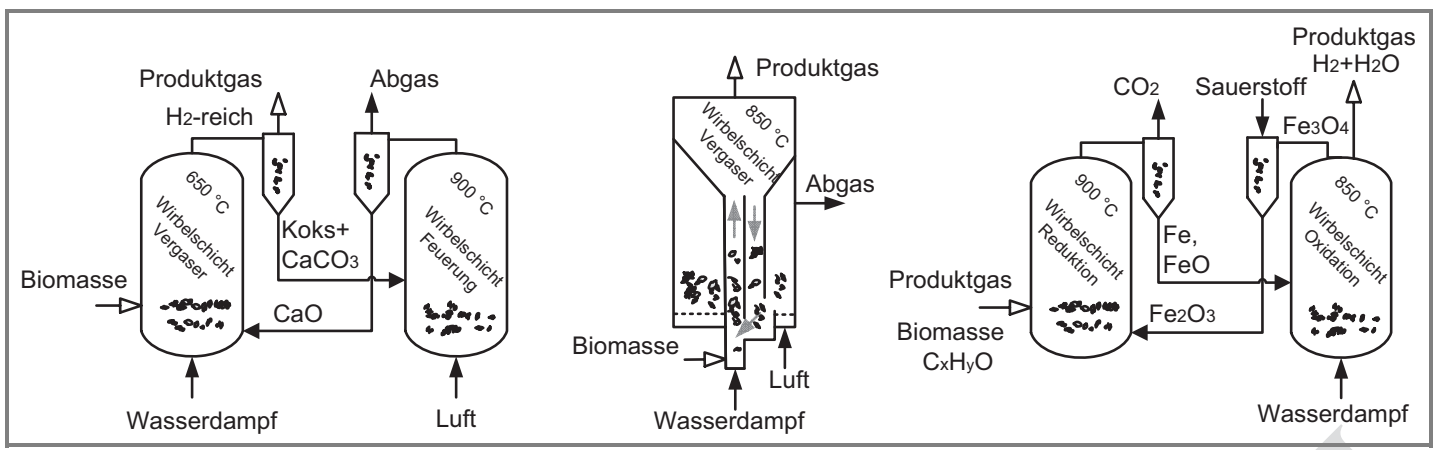

Abbildung 12. In Entwicklung befindliche Vergaserkonzepte. Links: Absorption Enhanced Reforming (AER), Mitte: Milena Vergaser, rechts: Coal Direct Chemical Looping Prozess.

Tabelle 8. Zusammensetzung der Produktgasgemische bei verschiedenen Vergasungsverfahren $[3,15,54]$.

\begin{tabular}{|c|c|c|c|c|c|c|}
\hline & [Vol.-\% $\left.\%_{\mathrm{tr}}\right]$ & $\mathrm{H}_{2}$ & $\mathrm{CO}$ & $\mathrm{CH}_{4}$ & $\mathrm{CO}_{2}$ & $\mathrm{~N}_{2}$ \\
\hline Festbett & Luft & $10-23$ & $15-25$ & $2-6$ & $10-15$ & $40-55$ \\
\hline Flugstrom & Luft & $7-15$ & 20 & 0 & 11 & 46 \\
\hline Flugstrom & Sauerstoff & 27 & 50 & 0 & & 4 \\
\hline Wirbelschicht & Luft/20 bar & 12 & 18 & 17 & 6 & 50 \\
\hline $\begin{array}{l}\text { FICFB- } \\
\text { Wirbelschicht }\end{array}$ & Dampf, allotherm & 40 & 25 & & 20 & 4 \\
\hline $\begin{array}{l}\text { AER- } \\
\text { Wirbelschicht }\end{array}$ & $\begin{array}{l}\text { Dampf, } \mathrm{CaO} \text {, } \\
\text { allotherm }\end{array}$ & $68-78$ & 4 & 12 & 10 & 4 \\
\hline $\begin{array}{l}\text { HPR- } \\
\text { Wirbelschicht }\end{array}$ & Dampf, allotherm & $40-50$ & $15-25$ & $5-10$ & $15-25$ & $5-10$ \\
\hline
\end{tabular}

Biomasseheizwerke und kleinere Biomasseheizkraftwerke zur Wärmeversorgung von Nahwärmenetzen, wie sie in Mitteleuropa sehr üblich sind, beschränken sich zukünftig entweder auf kleinere dezentrale Systeme $(100-2000 \mathrm{~kW}) \mathrm{mit}$ hohen lokalen Wärmeabnahmedichten und sehr kurzen Nahwärmenetzen oder auf Gebiete mit überdurchschnittlichen Wärmebedarfsdichten wie beispielsweise Tourismusgebiete oder Industrieprozesse. Hier ist auch der wärmegeführte Betrieb von Heizkraftwerken sinnvoll. Effizienzsteigerungen durch den Einsatz von Abgaskondensationsanlagen sind möglich. Für rein ländlich orientierte Wohngebiete ergibt sich aufgrund des mittlerweile sehr geringen Heizenergieverbrauches moderner Gebäude auf Basis fester Biomasse oftmals keine ökonomisch sinnvolle Möglichkeit.

Neben den ökonomischen Anforderungen werden in der Öffentlichkeit die ökologischen Aspekte sehr genau hinterfragt. Die Emissionen an Kohlenwasserstoffen und Feinstäuben und die Gewinnung der Biomasse stehen dabei im Mittelpunkt. Mittlerweile scheitern insbesondere kleinere kommunale Biomasseprojekte immer öfter auch aus diesen Gründen. Bei der zukünftigen dezentralen Biomassenutzung muss daher noch stärker auf die Nachhaltigkeit und die Reduzierung von Emissionen geachtet werden.

Die Biomassenutzung in dicht besiedelten Gebieten mit geringen lokalen Emissionen ist mit gasförmigen Produk- ten möglich. Mittelfristig wird daher erwartet, dass die dezentrale thermochemische Vergasung und die Aufbereitung des Gasgemischs zu einem ins bestehende Erdgasnetz einspeisefähigen Gas (SNG) an Bedeutung gewinnen. Mehrere Projekte in Österreich, Schweden und Deutschland befinden sich dazu bereits in der Realisierung. Die dezentrale und äußerst emissionsarme Nutzung in kleinen KWK-Anlagen, die möglichst effiziente Verstromung in GuD-Anlagen oder die Nutzung in Gasautos wird so ermöglicht. Die Lenkung der Nutzung erfolgt allerdings durch die Bedingungen von Förderprogrammen, wie z. B. dem Erneuerbare-Energien-Gesetz EEG [67]. Beispielsweise besteht für die Nutzung von Gasen biogenen Ursprungs in Gasautos derzeit in Deutschland keine Nachfrage.

Für Großanlagen zur Erzeugung von Kraftstoffen aus Biomasse (BtL) mit mehreren $100 \mathrm{MW}$ Leistung ist $\mathrm{zu}$ erwarten, dass eine Realisierung aufgrund der limitierten Biomasse-Ressourcen in Deutschland eher schwierig ist.

Außerhalb Deutschlands werden die Mitverbrennung von fester Biomasse in Kohlekraftwerken und die Nutzung in größeren Biomasseheizkraftwerken weiter an Bedeutung gewinnen. Dabei wird die Biomasse zu erheblichen Anteilen importiert werden. Insbesondere größere Energiebversorgungsunternehmen $\mathbf{\square}$ check $\mathbf{\square}$ werden sich hier engagieren. In Deutschland selbst wird die Mitverbrennung von Biomasse im Rahmen des EEGs nicht gefördert und wird daher kaum angewandt.

Für die Verbrennung von Biomasse haben sich Unterschub-, Rost- und Wirbelschichtfeuerungen etabliert. Bei der thermochemischen Vergasung sind Wirbelschichtverfahren ebenfalls üblich. Festbettvergaser konnten sich bisher aus meist technischen Gründen nicht stärker durchsetzen. Zur Erzeugung von Synthesegas kann aufbereitete Biomassen auch in Flugstromvergasern eingesetzt werden. 
Zusammenfassend lässt sich feststellen, dass sich die Möglichkeiten der thermischen Nutzung von Biomasse durch verschiedene Verfahrensentwicklungen erweitern. In Deutschland wird man aus strukturellen Gründen weiterhin dezentrale Lösungen bevorzugen, während andere Länder auch auf größere Kraftwerke, die Mitverbrennung und eine großtechnische Synthesegasherstellung setzen.

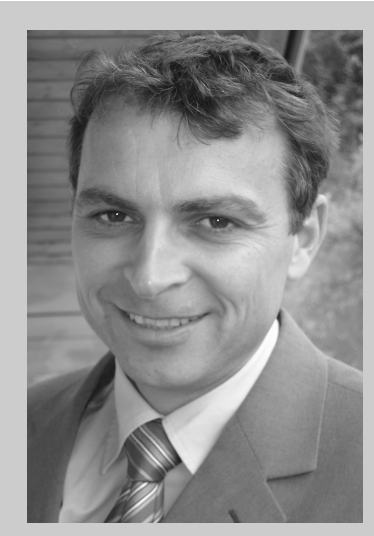

Matthias Gaderer, geb. 1968, studierte von 1991 1996 Verfahrenstechnik an der TU Graz und an der KTH Stockholm. 1997 bis 1999 war er als Verfahrensingenieur in Salzburg tätig. Im Anschluss arbeitete er bis 2007 am Bayerischen Zentrum für angewandte Energieforschung. Seit 2007 ist Dr. Garderer Mitarbeiter am Lehrstuhl Energiesysteme der Technischen Universität München, wo er im gleichen Jahr auch promovierte.

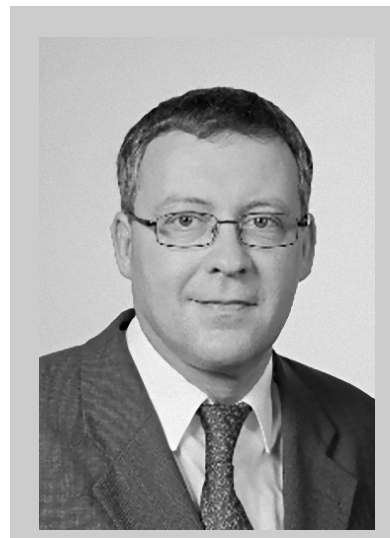

Hartmut Spliethoff schloss 1986 sein Maschinenbaustudium an der Universität Stuttgart ab und war dort von 1986-1991 als wissenschaftlicher Mitarbeiter am Institut für Verfahrenstechnik und Dampfkesselwesen beschäftigt. Nach der Promotion 1992 leitete er bis 1999 die Abteilung Dampferzeugung des Instituts. Prof. Spliethoff habilitierte sich 1999 in Verbrennung und Kraftwerkstechnik an der Universität Stuttgart und war 2000 Ordinarius am Institute for Thermal Power Engineering an der University of Technology Delft/NL. Seit 2004 hat er den Lehrstuhl für Energiesysteme der Technischen Universität München inne. $\square$ bitte prüfen

\section{Literatur}

[1] A. Meyer, Die städtische Verbrennungsanstalt für Abfallstoffe von Bullerdeich in Hamburg, Deutsche Vierteljahrsschrift für öffentliche Gesundheitspflege 1897, 29 (3), 353 - 378.

[2] Key Issues Q Mandates Secure Q Reliable Energy Supplies-History of U.S. Coal Use, National Energy Technology Laboratory, Pittsburgh, PA. www.netl.doe.gov/KeyIssues/historyofcoaluse.html

[3] H. Spliethoff, Power Generation from Solid Fuels, Springer, Heidelberg 2010.
[4] Key World Energy Statistcs, International Energy Agency, Paris 2008. www.iea.org/textbase/nppdf/free/2008/ key_stats_2008.pdf

[5 World Energy Outlook 2009, International Energy Agency, Paris 2010. www.iea.org/textbase/nppdf/free/2009/WEO2009.pdf

[6] Energiedaten, Bundesministerium für Wirtschaft und Technologie, Berlin 2011. www.bmwi.de/BMWi/Navigation/Energie/ Statistik-und-Prognosen/energiedaten.html

[7] Directive 2009/28/EC, European Parliament and Council, Brussels 2009.

[8] Verordnung über die Erzeugung von Strom aus Biomasse (Biomasseverordnung), Bundesministerium für Umwelt, Naturschutz und Reaktorsicherheit, Berlin 2001

[9] DIN EN 14961 Teil 1 bis 6, Feste Biobrennstoffe-Brennstoffspezifikationen, Beuth, Berlin 2010.

[10] Klärschlammverordnung (AbfKlärV), Bundesministerium für Umwelt, Naturschutz und Reaktorsicherheit, Berlin 1992.

[11] Verordnung über die umweltverträgliche Ablagerung von Siedlungsabfällen (Abfallablagerungsverordnung-AbfAblV), Bundesministerium für Umwelt, Naturschutz und Reaktorsicherheit, Berlin 2001.

[12] DIN 51730, Bestimmung des Ascheschmelzverhaltens, Beuth, Berlin 1998.

[13] W. Boie, Vom Brennstoff zum Rauchgas, Feuerungstechnisches Rechnen mit Brennstoffkenngrössen und seine Vereinfachung mit Mitteln der Statistik, Teubner (in Verwaltung), Wiesbaden 1957.

[14] A. Nordin et al., PhD Thesis, Umeå University, Sweden 1993.

[15] M. Kaltschmitt, H. Hartmann, H. Hofbauer, Energie aus Biomasse, 2nd ed., Springer, Berlin 2009.

[16] B. Sander, Biomass Bioenergy 1997, 12 (3), 177-183.

[17] I. Obernberger, F. Biedermann, Fraktionierte Schwermetallabscheidung in Biomasseheizwerken, Forschungsprojekt Fractio, Bios Bioenergiesysteme GmbH, Graz 1995.

[18] A Review of the Current State of Bioenergy Development in G8+5 Countries, GBEP (Global Bioenergy Partnership) Secretariat, Food and Agriculture Organization of the United Nations (FAO), Rome 2008.

[19] D. Thrän et al., Sustainable Strategies for Biomass use in the European Context. Analyses in the charged debate on national guidelines and the competition between solid, liquid and gaseous biofuels, Report 1/2006, Institut für Energetik und Umwelt $\mathrm{GmbH}$, Leipzig, Leipzig 2006.

[20] Entwicklung Pelletproduktion Deutschland, Deutscher Energie Pelletverband, Berlin 2011. www.depv.de/startseite/ marktdaten/entwicklung-pelletproduktion/

[21] C. Schlagitweit, Internationale Ökoenergiekonferenz, Sand in Taufers, Italy, October 2009. www.biomasseverband.at/ uploads/tx_osfopage//mediendatenbank /root01/3.\%20Veranstaltungen/3.2\%20Tagung/Internationale\%20\%C3\%96koenergiekonferenz/ppt/13\%20Rakos.pdf

[22] F. Magelli et al., Biomass Bioenergy 2009, 33 (3), 434-441.

[23] Pro Pellets Austria: Produktionskapazität, Produktion und Verbrauch, Pro Pellets Austria, Wolfsgraben, Austria 2011. www.propellets.at $/ \mathrm{cms} / \mathrm{cms}$.php?pageName $=341$

[24] M. Antonietti, Hydrothermale Karbonisierung von Biomasse: $\mathrm{CO}_{2}$-Senke oder effektive Rohstoffquelle?, Vortrag, Max-PlankInstitut, Potsdam 2006. www.mpikg.mpg.de/pdf/KolloidChemie/Scripte/HTC-lehrerPotsdam2010.pdf

[25] F. Bergius, Naturwissenschaften 1928, 16 (1), 1-10. Đcheck Vol \& Seitel 
[26] F. Bergius, Chemical reactions under high pressure, Nobel Lecture, Stockholm 1932. http://nobelprize.org/nobel_prizes/ chemistry/laureates/1931/bergius-lecture.pdf

[27] P. C. A. Bergman, 14th European Biomass Conference Q Exhibition, Paris, October 2005. www.ecn.nl/docs/library/report/2005/ rx05180.pdf

[28] P. C. A. Bergman, Combined Torrefaction and Pelletisation: The TOP Process, Energy research Centre of the Netherlands (ECN), Petten, Netherlands 2005.

[29] P. C. A. Bergman, Torrefaction for biomass co-firing in existing coal-fired power stations, Energy research Centre of the Netherlands (ECN), Petten, Netherlands 2005.

[30] F. Richarts, Workshop Biokohle, Justus-Liebig-Universität Gießen, Februar 2010. http://fss.plone.uni-giessen.de/fss/fbz/ fb08/biologie/pflanzenoek/forschung/workshop/copy_of_ workshop/richards/file/Richarts_HTC\%20Workshop_ 22-02-10.pdf

[31] C. P. Kleinschmidt, Torrefaction Workshop, Graz Januar 2011. http://www.bioenergytrade.org/pastevents/graz.html, 2011

[32] P. A. Jensen, B. Sander, K. Dam-Johansen, Biomass Bioenergy 2001, 20 (6), 447-457. DOI: 10.1016/S0961-9534(01)00006-X

[33] T. Wittmann, Erdöl, Erdgas, Kohle 2010, 126 (5), Deite?

[34] Wood Pellets-The Bioenergy Feedstock Solution? Global market, players and trade to 2015, Pöyry Forest Industry Consulting, Cheam, UK 2009.

[35] M. Peksa-Blanchard et al., IEA Bioenergy Task 40, Global Wood Pellets Markets and Industry: Policy Drivers, Market Status and Raw Material Potential, 2007. www.bioenergytrade.org/down loads/ieatask40pelletandrawmaterialstudynov2007final.pdf

[36] R. Tarcon, IEA Workshop on Cofiring Biomass with Coal, Drax Power Station, Selby, January 2011. www.iea-coal.org.uk/ publishor/systemcomponent_view.asp?LogDocId= 82487\&PhyDocId $=7694$

[37] Co-Firing-Database, IEA Bioenergy Task 32. www.ieabcc.nl

[38] B. Kreutzkam, C. Wieland, G. Balan, S. Gleis, H. Spliethoff, Abschätzung der Möglichkeiten und Grenzen zur Mitverbrennung biogener Brennstoffe in einem Steinkohlekessel, TK Verlag-ThoméKozmiensky, Neuruppin 2009, 136-145.

[39] T. Nussbaumer, Teil 1: Grundlagen der Holzverbrennung, Schweizer Baudokumentation, Blauen 2000. www.verenum.ch /publikationen.html

[40] D. Hein, T. Fischer, in VDI-GET-Tagung Thermische Nutzung fester Biomasse, Bericht 1588, VDI-Verlag Düsseldorf 2001.

[41] M. Gaderer, Dissertation, Technische Universität München 2007.

[42] M. Gaderer, F. Volz, R. Kunde, in Handbook of Combustion, Vol. 4, Wiley-VCH, Weinheim 2010, 141-169.

[43] 1. Verordnung zur Durchführung des Bundes-Immissionsschutzgesetzes (Verordnung über kleine und mittlere Feuerungsanlagen -1 . BImSchV), Bundesministerium für Umwelt, Naturschutz und Reaktorsicherheit, Berlin 2010.

[44] Verordnung über Anforderungen an die Verwertung und Beseitigung von Altholz, Altholzverordnung - AltholzV, Bundesministerium für Umwelt, Naturschutz und Reaktorsicherheit, Berlin 2002.

[45] Verordnung über genehmigungsbedürftige Anlagen - 4.BImSchV, Bundesministerium für Umwelt, Naturschutz und Reaktorsicherheit, Bonn 1985.

[46] Erste Allgemeine Verwaltungsvorschrift zum Bundes-Immissionsschutzgesetz, Technische Anleitung zur Reinhaltung der Luft - TA Luft, Bundesministerium für Umwelt, Naturschutz und Reaktorsicherheit, Berlin 2002.
[47] Verordnung über Großfeuerungs- und Gasturbinenanlagen 13. BImSchV, Bundesministerium für Umwelt, Naturschutz und Reaktorsicherheit, Berlin 2004.

[48] Verordnung über die Verbrennung und die Mitverbrennung von Abfällen - 17. BImSchV, Bundesministerium für Umwelt, Naturschutz und Reaktorsicherheit, Berlin 2003.

[49] V. Schmatloch, T. Brzovic, T. Bleul, R. Bolliger, in Proc. 10. Holzenergie-Symposium (Ed: T. Nussbaumer) ETH-Zürich, Zürich 2008. Wcheck

[50] W. Fritz, H. Kern, Reinigung von Abgasen, 2nd ed., Verlag Vogel, Würzburg 1990.

[51] R. Kunde, F. Volz, M. Gaderer, H. Spliethoff, BWK 2009, 61 (1), 58-66.

[52] R. Kunde, M. Adeili, F. Volz, M. Gaderer, 19th European Biomass Conference, Berlin, June 2011

[53] P. C. A. Bergman, A. R. Boersma, J. H. A. Kiel, M. J. Prins, K. J. Ptasinski, F. J. J. G. Janssen, Torrefaction for entrained flow gasification of biomass, ECN report C-05-067, 2005. www.ecn.nl/ docs/library/report/2005/c05067.pdf

[54] J. Karl, Dezentrale Energiesysteme, Oldenbourg-Verlag, München 2004.

[55] S. Bengtsson, Biomass Bioenergy, 2011, in press. DOI: 10.1016/ j.biombioe.2011.03.034

[56] K. Stahl, M. Neergard, in Proc. of the 10th Int. Conf. Biomass for Energy, 1998, 291-294. םVerlag?

[57] E. Kurkela, M. Kurkela, Advanced Biomass Gasification for HighEfficiency Power, Final Activity Report of BiGPower Project, VTT, 2009.

[58] N. Dahmen, E. Dinjus, Chem. Ing. Tech. 2010, 82 (8), $1147-$ 1152.

[59] The Carbo-V@ Process, Choren Industries, Freiberg.

[60] C. M. Van der Meijden, H. J. Veringa, L. P. L. M. Rabou, Biomass Bioenergy 2010, 34, 302-311.

[61] M. A. Paisley, R. P. Overend, 12th European Conference and Technology Exhibition on Biomass for Energy, Amsterdam, June 2002.

[62] H. Hofbauer et al., Developments in Thermochemical Biomass Conversion, The FICFB next term-gasification process, (Eds: A. V. Bridgwater, D. G. B. Boocock), Vol. 2, Dheck Blackie, London 1997, 1016-1025.

[63] T. Marquard-Möllenstedt, B. Stürmer, U. Zuberbühler M. Specht, in Encyclopedia of Electrochemical Power Sources, Elsevier, Amsterdam 2009, 249-258.

[64] C. M. Van der Meijden, A. Van der Drift, B. J. Vreugdenhil, 15th European Biomass Conference, Berlin, May 2007. www.milenatechnology.com/fileadmin/milenatechnology/ user/documents/reports/Milena_Berlin_2007_paper.pdf

[65] C. M. Van der Meijden, P. C. A. Bergman, A. Van der Drift, B. J. Vreugdenhil, 18th European Biomass Conference and Exhibition, Lyon, May 2010. www.milenatechnology.com/filead$\mathrm{min} /$ milenatechnology/user/documents/reports/Paper_ Preparations_for_a_10_MWth_Bio-CHP_demonstration_ based_on_the_MILENA_gasification_technology.pdf

[66] L. Fanxing, K. Hyung, D. Sridhar, L. Zeng, F. Wang, A. Tong, L.-S. Fan, Coal Direct chemical Looping (CDCL) process for hydrogen and power generation, The Ohio State University, 2009.

[67] Gesetz für den Vorrang Erneuerbarer Energien (Erneuerbare-Energien-Gesetz), EEG-Novelle 2012. http://www.eeg-aktuell.de/ 口genauer; was dort? 
DOI: $10.1002 /$ cite.201100132

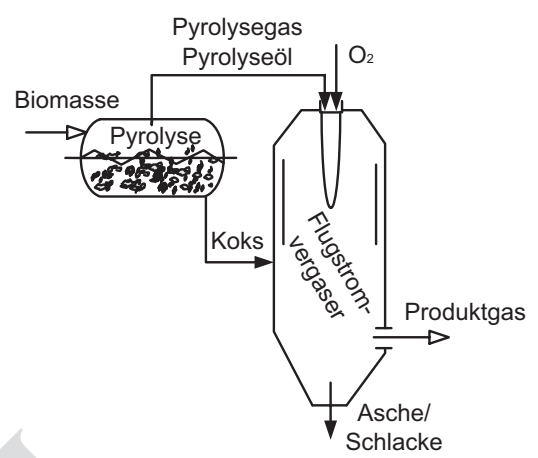

Thermische Nutzung von Biomasse und Reststoffen in Deutschland M. Gaderer*, H. Spliethoff

Eine Übersicht zum Stand der Technik und neue Entwicklungen auf dem Gebiet der thermischen Nutzung der Biomasse werden aufgezeigt. Die thermische Nutzung von Biomasse und Reststoffen ist eine wichtige Säule der Bereitstellung von Erneuerbaren Energien. Mit neuen Verfahren der Biomasseaufbereitung und Vorbehandlung können weitere Ressourcen zur Nutzung durch erschlossen werden. 